OPEN ACCESS

Edited by:

Kuo-Feng Hua,

National Ilan University, Taiwan

Reviewed by:

Joo Young Lee,

Catholic University of Korea,

South Korea

Lih-Chyang Chen,

Mackay Medical College, Taiwan

${ }^{*}$ Correspondence:

Svetlana F. Khaiboullina

sv.khaiboullina@gmail.com

Specialty section: This article was submitted to Inflammation,

a section of the journal

Frontiers in Immunology

Received: 18 September 2020 Accepted: 17 December 2020

Published: 03 February 2021

Citation:

Tezcan G, Garanina EE, Alsaadi M, Gilazieva ZE, Martinova EV,

Markelova MI, Arkhipova SS,

Hamza S, Mclntyre A, Rizvanov AA and Khaiboullina SF (2021)

Therapeutic Potential of Pharmacological Targeting NLRP3 Inflammasome Complex in Cancer.

Front. Immunol. 11:607881. doi: 10.3389/fimmu.2020.607881

\section{Therapeutic Potential of Pharmacological Targeting NLRP3 Inflammasome Complex in Cancer}

\author{
Gulcin Tezcan ${ }^{1,2}$, Ekaterina E. Garanina ${ }^{1}$, Mohammad Alsaadi ${ }^{1}$, Zarema E. Gilazieva ${ }^{1}$, \\ Ekaterina V. Martinova ${ }^{1}$, Maria I. Markelova ${ }^{1}$, Svetlana S. Arkhipova ${ }^{1}$, Shaimaa Hamza ${ }^{1}$, \\ Alan McIntyre ${ }^{3}$, Albert A. Rizvanov ${ }^{1}$ and Svetlana F. Khaiboullina ${ }^{1,4 *}$ \\ 1 Institute of Fundamental Medicine and Biology, Kazan Federal University, Kazan, Russia, ${ }^{2}$ Department of Fundamental \\ Sciences, Faculty of Dentistry, Bursa Uludag University, Bursa, Turkey, ${ }^{3}$ Centre for Cancer Sciences, Faculty of Medicine \\ and Health Sciences, University of Nottingham, Nottingham, United Kingdom, ${ }^{4}$ Department of Microbiology and \\ Immunology, University of Nevada, Reno, NV, United States
}

Introduction: Dysregulation of NLRP3 inflammasome complex formation can promote chronic inflammation by increased release of IL-1 $\beta$. However, the effect of NLRP3 complex formation on tumor progression remains controversial. Therefore, we sought to determine the effect of NLRP3 modulation on the growth of the different types of cancer cells, derived from lung, breast, and prostate cancers as well as neuroblastoma and glioblastoma in-vitro.

Method: The effect of Caspase 1 inhibitor (VX765) and combination of LPS/Nigericin on NLRP3 inflammasome activity was analyzed in A549 (lung cancer), MCF-7 (breast cancer), PC3 (prostate cancer), SH-SY5Y (neuroblastoma), and U138MG (glioblastoma) cells. Human fibroblasts were used as control cells. The effect of VX765 and LPS/Nigericin on NLRP3 expression was analyzed using western blot, while IL-1 $\beta$ and IL-18 secretion was detected by ELISA. Tumor cell viability and progression were determined using Annexin $\mathrm{V}$, cell proliferation assay, LDH assay, sphere formation assay, transmission electron microscopy, and a multiplex cytokine assay. Also, angiogenesis was investigated by a tube formation assay. VEGF and MMPs secretion were detected by ELISA and a multiplex assay, respectively. Statistical analysis was done using one-way ANOVA with Tukey's analyses and Kruskal-Wallis one-way analysis of variance.

Results: LPS/Nigericin increased NRLP3 protein expression as well as IL-1 $\beta$ and IL-18 secretion in PC3 and U138MG cells compared to A549, MCF7, SH-SY5Y cells, and fibroblasts. In contrast, MIF expression was commonly found upregulated in A549, PC3, SH-SY5Y, and U138MG cells and fibroblasts after Nigericin treatment. Nigericin and a combination of LPS/Nigericin decreased the cell viability and proliferation. Also, LPS/ Nigericin significantly increased tumorsphere size in PC3 and U138MG cells. In contrast, the sphere size was reduced in MCF7 and SH-SY5Y cells treated with LPS/Nigericin, while no effect was detected in A549 cells. VX765 increased secretion of CCL24 in A549, MCF7, PC3, and fibroblasts as well as CCL11 and CCL26 in SH-SY5Y cells. Also, VX765 
significantly increased the production of VEGF and MMPs and stimulated angiogenesis in all tumor cell lines.

Discussion: Our data suggest that NLRP3 activation using Nigericin could be a novel therapeutic approach to control the growth of tumors producing a low level of IL-1 $\beta$ and IL-18.

Keywords: NLRP3, inflammasome, cancer, IL-1 $\beta$, nigericin

\section{INTRODUCTION}

A chronic inflammatory microenvironment is one of the predisposing factors that can stimulate malignant transformation (1). The tumor microenvironment contains diverse inflammatory constituents, leukocytes, pro-inflammation cytokines and tumor cells (2), which can promote angiogenesis, tumor growth and metastasis (2). The inflammasome, an intracellular oligomeric protein complex, plays a key role in the regulation of inflammation (3). Inflammasomes are activated in response to pathogen-associated molecular patterns (PAMPs) and damageassociated molecular patterns (DAMPs) released from infected cells, damaged tissues and tumors (4). PAMPs and DAMPs bind to Toll-like receptors (TLR) and trigger the expression of proinflammatory IL-1 $\beta$ and IL-18 cytokines (5). The most characterized inflammasome, Nucleotide-binding domain Leucine-rich Repeat (NLR) and Pyrin domain containing receptor 3 (NLRP3), is tightly regulated in resting cells (6). However, altered expression of NLRP3 was found in several pathological conditions, including cancer (6).

NLRP3 requires two signals: priming and activation (7). Binding of PAMPs and DAMPs to TLRs prime the cells and activate NLRP3 and Pro-Caspase 1 transcription $(6,8)$. Danger associated ligands such as pore formation and potassium $(\mathrm{K}+)$ efflux (9, 10), lysosomal destabilization/rupture $(11,12)$ and mitochondrial reactive oxygen species release (ROS) (13) provide the second signal promoting the assembly of an adaptor, apoptosis-associated speck-like protein containing a CARD (ASC) and Pro-Caspase 1 to form a functional inflammasome complex $(14,15)$. The inflammasome then cleaves the Caspase 1 which, subsequently releases active IL-1 $\beta$ and IL-18 (16).

The role of inflammasomes was extensively investigated in macrophages and dendritic cells (17). However, recently it was demonstrated that NLRP3 activation is not an exclusive feature of immune cells, and it was also detected in tumor cells (18-21), while its role in tumorigenesis remains controversial. Some reports indicate that aberrant activation of inflammasomes promotes carcinogenesis and maintains the malignant microenvironment in breast cancer, fibrosarcoma, gastric carcinoma, and lung metastasis (22-24). In contrast, the anti-cancer effect of inflammasomes via induction of pyroptosis and activation of the immune response was shown to protect against colorectal cancer (25).

Recently, multiple inhibitors and activators of the NLRP3 inflammasome pathway were investigated as potential therapeutics for inflammasome-linked diseases $(26,27)$. However, the efficacy of inflammasome targeting treatment in cancers remains unclear, due to the conflicting results obtained using tumor cells. The anti-tumor activity of several compounds targeting NLRP3 inflammasomes was studied, including Nigericin and VX-765. Nigericin, a microbial toxin, activates NLRP3 by inducing $\mathrm{K}^{+}$efflux leading to the caspase-1 release and IL-1 $\beta$ secretion $(28,29)$. The anti-tumor effect of Nigericin was demonstrated in several carcinomas (30-34), however, it remains unknown whether the anti-tumor effect of Nigericin is linked to NLPR3. VX765 can block the caspase-1 ability to proteolytically cleave pro-IL-1 $\beta$ and pro-IL-1 $(26,35)$. Although recent studies demonstrated the potential therapeutic efficacy of VX765, its effect on tumor cells is largely unknown $(26,35,36)$.

Therefore, in this study, we sought to investigate in vitro effects of Nigericin and VX765 on multiple tumor cell types derived from a variety of cancers, including lung, breast, prostate, as well as neuroblastoma and glioblastoma. We found that tumors demonstrate different levels of NLRP3 inflammasome activation and IL-1 $\beta$ and IL-18 secretion. In tumor cell lines where NLRP3 activation and, IL-1 $\beta$ and IL-18 secretion are low, Nigericin demonstrated an anti-tumor effect. In contrast, in tumor cell lines where NLRP3 activation and, IL-1 $\beta$ and IL-18 secretion are high, although Nigericin triggers initial tumor cell death, cells recover and tumors remain active.

\section{METHODS}

\section{Cell Lines}

Cell lines, A549 (non-small-cell lung cancer cell line), MCF7 (breast adenocarcinoma cell line), PC3 (prostatic small-cellcarcinoma cell line), and SH-SY5Y (neuroblastoma cell line) were purchased from the American Type Culture Collection (ATCC; Rockville, USA). U138MG, a glioblastoma cell line was kindly provided by Prof. Dr. Berrin Tunca (Uludag University of Turkey). Cells were maintained in Dulbecco's modified Eagle medium (DMEM) supplemented with $10 \%$ fetal bovine serum (FBS, Atlanta Biologicals), $2 \mathrm{mM} \mathrm{L-glutamine,} 25 \mathrm{U} / \mathrm{ml}$ penicillin, and $25 \mu \mathrm{g} / \mathrm{ml}$ streptomycin. All cell lines were grown at $37^{\circ} \mathrm{C}$ in a humidified chamber supplemented with $5 \% \mathrm{CO}_{2}$.

\section{Primary Cell Isolation and Maintenance}

Primary fibroblast cells were isolated from human healthy skin biopsies which were collected after the cosmetic surgery. Skin fragments were crushed into small pieces (1-2 $\mathrm{mm})$ and 
incubated in DMEM Low Glucose Medium (PanEco, Moscow, Russia), supplemented with 10\% FBS (HYCLONE, Utah, USA), $50 \mathrm{U} / \mathrm{ml}$ of penicillin, $50 \mu \mathrm{g} / \mathrm{ml}$ of streptomycin (PanEco, Moscow, Russia), and $2 \mathrm{mM}$ L-glutamine for seven days in a $5 \% \mathrm{CO}_{2}$ humidified incubator at $37^{\circ} \mathrm{C}$. On day 7 , the culture medium was replaced, and cells were cultured for 16-21 days. Cells in their $4^{\text {th }}$ passage were used in the experiments.

HUVECs were isolated from umbilical cord vein as described previously [45-46]. Briefly, the umbilical vein was washed with Dulbecco's phosphate-buffered saline (DPBS) (PanEco, Moscow, Russia). The umbilical vein was subjected to enzymatic dissociation using 0.25\% trypsin-EDTA (PanEco, Moscow, Russia) for $20 \mathrm{~min}$ and detached endothelial cells were collected. Collected cells were stained with anti-CD31 (PECAM-1) (SC13537), anti-CD105 (Endoglin) (sc-18838), and anti-CD146 (MelCam) (sc-18837 PE) (all from Santa Cruz Biotechnology, CA) and analyzed using flow cytometry on FACS Aria III (Becton, Dickinson and Company, Becton Drive Franklin Lakes, NJ) to investigate their identity. Primary HUVEC cells were cultured in the standard medium supplemented with, 1\% of nonessential amino acids (Gibco, Life Technologies, MA, USA), $5 \mathrm{U} / \mathrm{ml}$ of heparin and $10 \mathrm{mg} / \mathrm{L}$ Endothelial cell growth supplement (Sigma, St. Louis, USA), $10 \mathrm{ng} / \mathrm{ml}$ FGF2, $10 \mathrm{ng} / \mathrm{ml}$ vascular endothelial growth factor (GenScript, NJ, USA), $10 \mathrm{ng} / \mathrm{ml}$ epithelial growth factor (GenScript, NJ, USA), $10 \mathrm{ng} / \mathrm{ml}$ insulin-like growth factor (GenScript, NJ, USA). Cells were maintained in a $5 \% \mathrm{CO}_{2}$ humidified incubator at $37^{\circ} \mathrm{C}$ and used up to the $4^{\text {th }}$ passage.

\section{Ethics Statement}

This study was done in accordance with the recommendations of the Biomedicine Ethics Expert Committee of Kazan (Volga region) Federal University, the Republic of Tatarstan, Russian Federation with written informed consent from all subjects. All subjects gave written informed consent in accordance with the Declaration of Helsinki. Human tissue sample collection was approved by the local Ethical Committee of Kazan (Volga region) Federal University based on article 20 of the Federal Legislation on "Health Protection of Citizens of the Russian Federation” № 323-FL, 21.11.2011. Signed informed consent was obtained from each donor.

\section{Western Blot}

Total protein extracts were prepared using Sodium dodecyl sulfate (SDS) reducing buffer (Biorad, CA, USA), separated on $8-12 \%$ gradient SDS polyacrylamide gels and transferred on Polyvinylidene difluoride (PVDF) membranes (Biorad, CA, USA). Membranes were blocked [Tris-buffered saline (TBS), $0.1 \%$ Tween $20,5 \% \mathrm{BSA}$ ] for $1 \mathrm{~h}$ followed by overnight incubation with the monoclonal rabbit anti-human NLRP3 (1:300, Invitrogen, IL, USA) antibody at $4^{\circ} \mathrm{C}$. Membranes were washed with TBS and $0.1 \%$ Tween 20 and incubated for $1 \mathrm{~h}$ at room temperature with anti-rabbit IgG (1:1,000, Santa Cruz Biotechnology, Germany) and mouse anti-human Actin BetaHRP conjugated $(1: 1,000$, Sigma) antibodies. Western blot results were visualized using Clarity Western ECL reagents (Biorad, CA, USA) and a ChemiDoc XRS + (Biorad, CA,
USA). Protein levels were quantified using NIH ImageJ software version $1.52 \mathrm{a}$.

\section{Enzyme-Linked Immunosorbent Assay}

Levels of IL-1 $\beta$, IL-18, and VEGF were measured using commercially available ELISAs (VECTOR-BEST, Novosibirsk, Russia). Each ELISA analysis was done in triplicate. To quantify IL-1 $\beta$, IL-18, and VEGF levels, the absorbance of samples at 490 and $680 \mathrm{~nm}$ were measured using TECAN Infinite 200 Pro fluorimeter (Grödig, Austria). The $680 \mathrm{~nm}$ absorbance (background signal from the instrument) value was subtracted from the $490 \mathrm{~nm}$ absorbance value.

\section{Annexin V Analysis}

Cell viability was assessed using APC Annexin V Apoptosis Detection Kit with Propidium Iodide (Sony Biotechnology, USA) according to manufacturer's protocol. Stained cells were immediately analyzed by flow cytometry using BD FACSAria III (BD Biosciences, USA) and data processed with FlowJo software package (FlowJo LLC, USA).

\section{Real-Time Cell Proliferation Assay}

The xCELLigence biosensor cell analysis system (ACEA Biosciences, USA) was used for the real-time monitoring of cell proliferation. Cells $\left(5 \times 10^{3}\right)$ were seeded in each well of Eplate 16 (ACEA Biosciences, USA) for $24 \mathrm{~h}$ and used to determine the cell index every $15 \mathrm{~min}$.

\section{Lactate Dehydrogenase Assay}

Cells $\left(2 \times 10^{4}\right.$ cells/well $)$ were plated in 96-well plates and incubated overnight $\left(37^{\circ} \mathrm{C}, \mathrm{CO}_{2}\right)$. The medium was removed, and cells were incubated with fresh medium containing VX765, lipopolysaccharide (LPS) and Nigericin for $24 \mathrm{~h}$. Medium $(50 \mu \mathrm{l})$ was collected and used for the LDH assays. Untreated cells were treated with the lysis solution for $45 \mathrm{~min}$ and used as a positive control to determine the maximum $\mathrm{LDH}$ release. The culture medium was used as a negative control. LDH released by cells into the medium was determined using the Pierce ${ }^{\mathrm{TM}} \mathrm{LDH}$ cytotoxicity assay kit (Thermo Scientific, Pierce Biotechnology, Rockford, IL, USA) according to the manufacturer's instructions. To quantify LDH activity, the absorbance of samples at 490 and $680 \mathrm{~nm}$ was measured using TECAN Infinite 200 Pro fluorometer (Grödig, Austria). The $680 \mathrm{~nm}$ absorbance (background signal from the instrument) value was subtracted from the $490 \mathrm{~nm}$ absorbance value. The cytotoxicity (\%) was calculated by (test sample - negative control)/(positive control negative control) $\times 100$.

\section{Sphere Formation Assay}

Plates (24 well) were pre-coated with $250 \mu$ of Matrigel (BD Biosciences, Franklin Lakes, NJ, USA) and incubated at $37^{\circ} \mathrm{C}$ for $30 \mathrm{~min}$. Tumor cells $\left(1 \times 10^{4}\right)$ were seeded onto the Matrigel-coated plate and maintained in the culture medium (5\% $\mathrm{CO}_{2}, 37^{\circ} \mathrm{C}$ ). After 5 days, tumorspheres were analyzed using a Zeiss Observer Z1 inverted microscope (Göttingen, Germany). The size of spheres was measured using Axiovision Rel 4.5 software (Göttingen, Germany). 


\section{JC-1 Staining to Detect Mitochondrial Membrane Potential $(\Delta \psi \mathrm{m})$}

Cell monolayers were washed with PBS and incubated with $8 \mu \mathrm{M}$ of JC-1 solution at $37^{\circ} \mathrm{C}$ for $20 \mathrm{~min}$. The supernatant was removed; cell monolayers were washed twice with PBS and incubated in medium for $24 \mathrm{~h}$ in $5 \% \mathrm{CO}_{2}$ before analyzing by flow cytometry using a BD FACSAria III (BD Biosciences, USA). Data were processed using FlowJo software package (FlowJo LLC, USA). The excitation wavelength was $490 \mathrm{~nm}$ at which the JC-1 monomer was detected, and the emitting wavelength was set to $530 \mathrm{~nm}$, at which the JC-1 polymer was detected.

\section{Cytokine Assay}

The Bio-Plex Pro ${ }^{\mathrm{TM}}$ Human Chemokine Panel, 40-Plex and BioPlex Pro ${ }^{\text {TM }}$ Human MMP Panel, 9-Plex were used to analyze samples according to the manufacturer's recommendations. Fifty microliters of sample were used for determining cytokine concentration and data was analyzed using a Luminex 200 analyzer with MasterPlex CT control software and MasterPlex QT analysis software (MiraiBio division of Hitachi Software San Francisco, CA, USA).

\section{Transmission Electron Microscopy}

Transmission electron microscopy (TEM) was done using $0.3 \times 10^{6}$ cells following a standard protocol. Briefly, cells were fixed in a $2.5 \%$ phosphate-buffered glutaraldehyde solution (Merck, Kenilworth, NJ, USA) at $4^{\circ} \mathrm{C}$ for $24 \mathrm{~h}$. Cells were then washed (2x PBS) and fixed with $1 \% \mathrm{OsO}_{4}$ (Electron Microscopy Sciences, Hatfield, USA) at room temperature for $1 \mathrm{~h}$, dehydrated using an ethanol gradient, and embedded in EPON (Electron Microscopy Sciences, Hatfield, USA). Ultrathin sections $(0,1 \mu \mathrm{m})$ were contrasted with uranyl acetate and lead citrate and examined using a transmission electron microscope (Hitachi HT7700, Tokyo, Japan).

\section{Endothelial Cell Tube Formation Assay}

96-well plates were pre-coated with $50 \mu \mathrm{l}$ of growth factorreduced Matrigel (BD Biosciences, Franklin Lakes, NJ, USA) and incubated at $37^{\circ} \mathrm{C}$ for $30 \mathrm{~min}$. HUVECs $\left(2 \times 10^{4}\right)$ were seeded in the Matrigel-coated wells and incubated with cell-free supernatants derived from VX765, LPS and Nigericin treated cells. VEGF $(10 \mathrm{ng} / \mathrm{ml})$ was used as a positive control. Tube morphology was analyzed at $24 \mathrm{~h}$ and two wells per group were counted. Tube numbers and branch length were measured and analyzed using NIH ImageJ software version 1.52a.

\section{Statistical Analyses}

Statistical analysis was done using the IDE RStudio for the $\mathrm{R}$ (version 3.6.0) software (RStudio, Boston, MA, USA). One-way ANOVA with Tukey's post hoc analysis was utilized to evaluate the findings of ELISA, Annexin V, JC1, and sphere formation assays, where the data were parametric. The Kruskal-Wallis oneway analysis of variance for comparisons between individual experimental groups was utilized for statistical analysis of the $\mathrm{LDH}$, Multiplex analyses and Tube formation assays, where the data were nonparametric. Data are presented as mean $\pm \mathrm{SE}$. Significance was established at a value of $\mathrm{p}<0.05$.

\section{RESULTS}

\section{Analysis of NLRP3 Activation and Inhibition After Nigericin and VX765 Treatment}

NLRP3 activation requires two signals: priming with lipopolysaccharide (LPS) followed by activation, using Nigericin or ATP (37). Therefore, to activate NLRP3 we treated all cancer cells and fibroblasts with LPS, followed by Nigericin. Nigericin and combination of LPS with Nigericin (LPS/Nigericin) increased NLRP3 protein expression in all tumor cell lines as compared to untreated tumor cells and in Nigericin and LPS/Nigericin treated fibroblasts as compared to untreated fibroblasts (Figures 1A-C and Supplementary Figures 1A-C). Additionally, production IL-1 $\beta$ and IL-18 and their levels in culture medium were increased in cells treated with the combination of LPS/Nigericin (Figures 1D-F, Supplementary Figures 1D-F). These data confirm that LPS/ Nigericin induces the formation of NLRP3 inflammasome complex in all tumor cell lines and the fibroblasts. After LPS/ Nigericin treatment, the IL- $1 \beta$ production was higher in U138MG (23.10 pg/ml cytosol vs $14.02 \mathrm{pg} / \mathrm{ml}$ medium) (Figure 1D) and PC3 (42.34 pg/ml cytosol vs $9.95 \mathrm{pg} / \mathrm{ml}$ medium) (Supplementary Figure 1D) cells compared to untreated controls. In addition, after LPS/Nigericin treatment, IL-1 $\beta$ production was higher in U138MG and PC3 cells compared to LPS/Nigericin treated Fibroblasts $(\mathrm{p}<0.001$ and $\mathrm{p}<0.001$ ). In fibroblasts, the IL- $1 \beta$ production was $9.16 \mathrm{pg} / \mathrm{ml}$ cytosol vs $14.26 \mathrm{pg} / \mathrm{ml}$ medium after LPS/Nigericin treatment (Figure 1F, Table 1). In contrast, IL-1 $\beta$ production and secretion were the lowest in SH-SY5Y cells incubated with LPS/Nigericin (IL-1 $3: 3.77 \mathrm{pg} / \mathrm{ml}$ cytosol vs $10.93 \mathrm{pg} / \mathrm{ml}$ medium) (Figure 1E). IL-1 $\beta$ production in SH-SY5Y cells was significantly lower than in fibroblasts (IL-1 $\beta: 9.16 \mathrm{pg} / \mathrm{ml}$ cytosol vs $14.26 \mathrm{pg} / \mathrm{ml}$ medium) ( $\mathrm{p}<0.001$ and $\mathrm{p}<0.001)$. Additionally, although, IL-1 $\beta$ secretion was induced in LPS/Nigericin treated MCF7 (11.89 pg/ml cytosol vs $7.85 \mathrm{pg} / \mathrm{ml}$ medium) (Supplementary Figure 1E) and A549 cells $(9.36 \mathrm{pg} / \mathrm{ml}$ cytosol vs $7.67 \mathrm{pg} / \mathrm{ml}$ medium) (Supplementary Figure 1F), when compared to untreated MCF7 and A549 cells, the production of IL-1 $\beta$ was lower than in LPS/Nigericin treated U138MG and PC3 cells.

Similar to IL-1 $\beta$, IL-18 production and secretion were increased in all tumor cell lines treated with LPS/Nigericin compared to untreated control (Table 1). IL-18 production was higher in PC3 cells $(13.83 \mathrm{pg} / \mathrm{ml}$ cytosol vs $11.40 \mathrm{pg} / \mathrm{ml}$ medium) (Supplementary Figure 1D) compared to LPS/ Nigericin treated fibroblasts $(10.96 \mathrm{pg} / \mathrm{ml}$ cytosol vs $7.10 \mathrm{pg} / \mathrm{ml}$ medium) ( $\mathrm{p}<0.001$ and $\mathrm{p}<0.001)$. In contrast, LPS/Nigericin leaded lower IL-18 production and release in SH-SY5Y (7.26 pg/ $\mathrm{ml}$ cytosol vs $7.81 \mathrm{pg} / \mathrm{ml}$ medium) (Figure 1E), MCF7 (7.29 pg/ $\mathrm{ml}$ cytosol vs $6.88 \mathrm{pg} / \mathrm{ml}$ medium) (Supplementary Figure 1E) and A549 cells (10.39 pg/ml cytosol vs $5.52 \mathrm{pg} / \mathrm{ml}$ medium) 
A

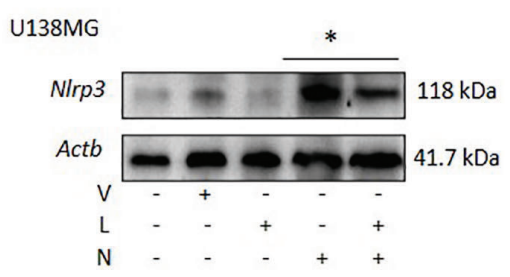

B SH-SY5Y

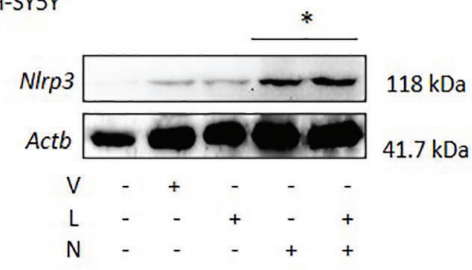

C

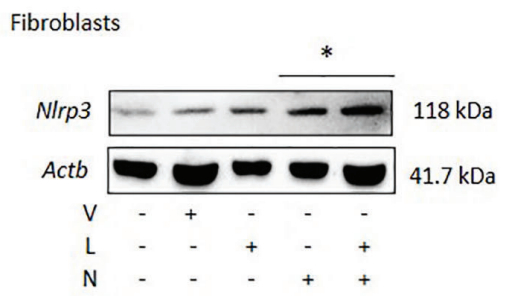

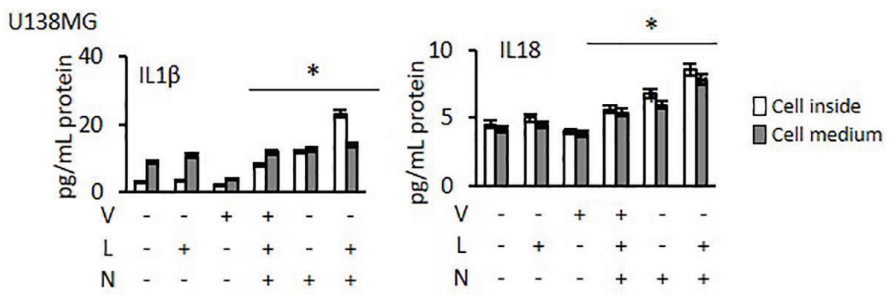

E SH-SY5Y
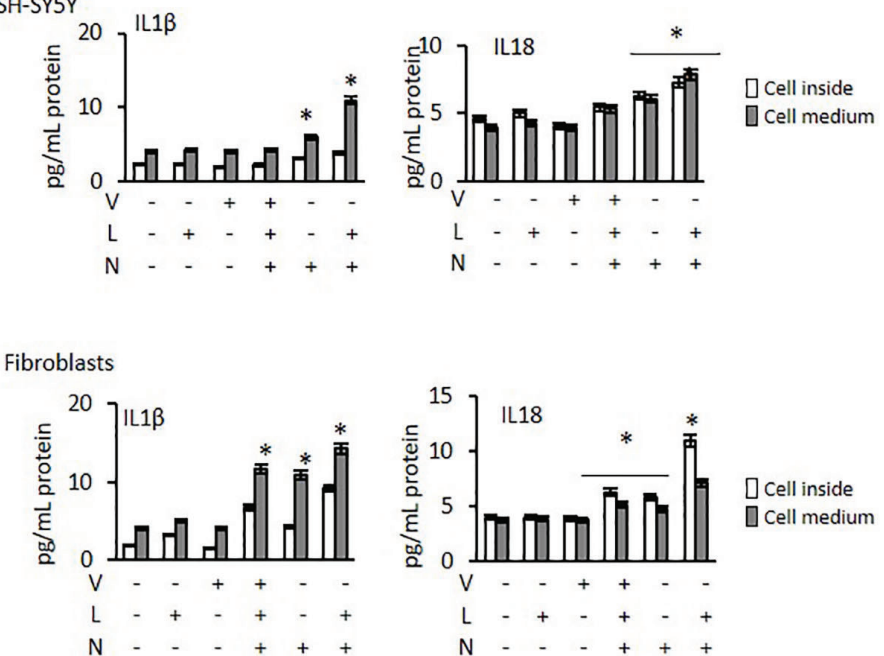

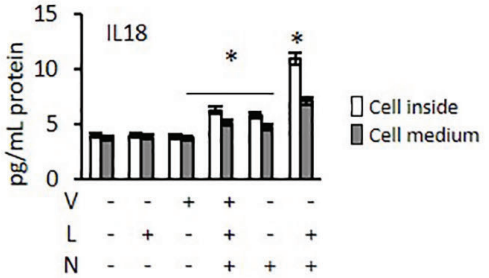

FIGURE 1 | Effect of NLRP3 modulation in U138MG and SH-SY5Y cells, where one of the highest and one of the lowest NLRP3 expression was observed and in Fibroblasts: Nigericin $(20 \mu \mathrm{M}$, Invivogen) treatment for $24 \mathrm{~h}$ with and without $3 \mathrm{~h}$ pre-incubation with LPS $(1 \mu \mathrm{g} / \mathrm{ml}$, Sigma, St. Louis, USA) was used to activate the NLRP3 inflammasome. To inhibit Caspase 1, cells were treated with VX765 (20 $\mu \mathrm{M}$, Invivogen). (A-C) NLRP3 protein expression was demonstrated by western blot. (D-F) IL-1 $\beta$ and IL-18 levels were quantified, from inside the cells and in the medium representing levels secreted from the cells, by ELISA. the cells by ELISA.

U, Untreated; V, VX765; L, LPS; N, Nigericin; LN, LPS/Nigericin. * $p<0.05, n=3$.

(Supplementary Figure 1F) compared to similarly treated fibroblasts $(\mathrm{p}<0.001)$. Interestingly, there were no statistically significant differences in IL-18 production in U138MG $(8.57 \mathrm{pg} /$ $\mathrm{ml}$ cytosol vs $7.80 \mathrm{pg} / \mathrm{ml}$ medium) (Figure 1D), SH-SY5Y cells
(7.26 pg/ml cytosol vs $7.81 \mathrm{pg} / \mathrm{ml}$ medium), and fibroblasts (10.96 pg/ml cytosol vs $7.10 \mathrm{pg} / \mathrm{ml}$ medium) after LPS/ Nigericin treatment (Figure 1F, Table 1). Collectively, our findings demonstrate that IL- $1 \beta$ production is a more robust

TABLE 1 | Summary of the significantly altered cytokines in tumor cells and fibroblasts following NLRP3 modulation (compared to untreated controls) $(p<0.05$, full data available in Supplementary Table 4).

\begin{tabular}{|c|c|c|c|c|}
\hline & vX765 & LPS & Nigericin & LPS\& Nigericin \\
\hline & & $\uparrow G M C S F$ & $\uparrow \mathrm{MIF}$ & $\uparrow G M C S F$, MIF \\
\hline PC3 & - & - & $\uparrow \mathrm{MIF}$ & $\begin{array}{l}\text { †CCL21, CCL11, GMCSF, CCL1, IL4, CCL8, } \\
\text { CCL22, CXCL9, CCL3, CCL23, CCL17, TNFa }\end{array}$ \\
\hline U138MG & - & - & $\uparrow \mathrm{MIF}$ & $\uparrow \mathrm{MIF}$ \\
\hline
\end{tabular}

Fibroblasts - $\quad \uparrow C C L 21$, CXCL13, CCL27, CXCL5, CCL11, CCL24, CCL26, GMCSF, CXCL1, $\uparrow M$ MIF CXCL2, CCL1, IL2, IL4, IL6, CXCL8, IL10, IL16, CXCL10, CXCL11, CCL2, CCL8, CCL7, CCL22, CXCL9, CCL5, CCL20, CCL19, CCL23, CXCL16, CXCL12, CCL17, CCL25 
indicator than IL-18, of NLRP3 activation across the panel of cells investigated here. The NLRP3 activation ability and IL-1 $\beta$, IL-18 secretions were also confirmed after treatment with LPS/ ATP in PC3 (one of the high NLRP3 expressed cell lines) and MCF7 (one of the low NLRP3 expressed cell lines) cells. After LPS/ATP treatment, the expression levels of NLRP3 were similar to that of cells after LPS/Nigericin treatment (Supplementary Figure 2).

VX765 as a single agent did not significantly affect NLRP3 protein expression (Figures 1A-C, Supplementary Figures 1A-C) in any cell lines. When cells were treated with VX765, the effect of LPS/Nigericin on IL-1 $\beta$ and IL-18 release was significantly reduced in most of the cell lines investigated ( $p<0.05$; Figures 1D-F, Supplementary Figures 1D-F, Supplementary Table 1). As an inflammasome inhibitor, the effect of VX765 was confirmed by another NLRP3 inhibitor, Glybenclamide in PC3 and MCF7 cells (Supplementary Figure 2). These data suggest that VX765 did not affect on NLRP3 expression but has an indirect mechanism of action of inflammasome inhibition, potentially via hindering Caspase 1 activity whereas Glybenclamide directly suppressed NLRP3 expression.

\section{Nigericin Inhibits, While VX765 Stimulates Tumor Cell Viability and Proliferation}

The effect of Caspase 1 inhibition and NLRP3 activation on cell viability was demonstrated using Annexin-V-FITC/PI assay. Cells demonstrate both, Annexin V and PI when nonapoptotic programmed cell deaths is induced (38). Tumor cell lines and fibroblasts were treated with VX765, LPS, Nigericin and LPS/Nigericin. VX765 had a limited effect on the percentage of Annexin V and PI positive cells in all cell lines ( $p>0.05$, Figure 2, Supplementary Figure 3, Supplementary Table 2). LPS decreased the percentage of PC3 and SH-SY5Y cells positive for Annexin V and PI compared to untreated controls (PC3 and SH-SY5Y: $p<0.05$, Figures 2A, B), while the number of Annexin $\mathrm{V}$ and PI positive A549, MCF7 and U138MG cells were not affected (A549, MCF7, and U138MG: p > 0.05, Supplementary Figures 2A, B). LPS increased the percentage of fibroblasts positive for Annexin V \& PI compared to untreated controls ( $\mathrm{p}=0.013$, Figure 2C).

Treatment with Nigericin alone significantly increased the percentage of Annexin V and PI positive cells in all cell lines compared to untreated controls except MCF7 and fibroblasts ( $\mathrm{p}<0.05$ ). (Figure 2, Supplementary Figure 2). Similarly, LPS/ Nigericin significantly increased the percentage of Annexin V and PI positive cells in all cell lines ( $\mathrm{p}<0.001$, Figure 2 , Supplementary Figure 3, Supplementary Table 2). The effect of Nigericin and LPS/Nigericin on PC3 cells, where NLRP3 expression was the highest, and SH-SY5Y, where NLRP3 activation was the lowest is shown in Figures 2A, B. The percentage of Annexin V \& PI positive cells after Nigericin and LPS/Nigericin treatments were significantly higher in PC3 cells compared to fibroblasts ( $\mathrm{p}<0.001$ and $\mathrm{p}<0.001$ ). In addition, although the percentage of Annexin V \& PI cells in Nigericin and LPS/Nigericin treated SH-SY5Y cells was higher than in fibroblasts $(\mathrm{p}<0.001$ and $\mathrm{p}<0.001)$, it was significantly lower than in equivalently treated PC3 cells ( $\mathrm{p}<0.001$ and $\mathrm{p}<0.001)$. In U138MG cells, where Nigericin and LPS/Nigericin induced one of the highest NLRP3 expressions, the percentage of Annexin V \& PI cells was significantly higher than in equivalently treated fibroblasts $(\mathrm{p}<0.001$ and $\mathrm{p}<0.001)$. Similarly, in MCF7 and in A549 cells, where Nigericin and LPS/Nigericin induced low inflammasome expression, the percentage of Annexin V \& PI cells was significantly higher compared to equivalently treated fibroblasts. It should be noted that the percentage of Annexin V \& PI cells in MCF7 and A549 cells was lower than in equivalently treated PC3 and U138MG cells. The effect of Nigericin and LPS/Nigericin on U138MG, MCF7, and A549 cell vitality is shown in Supplementary Figures 3 A-C. The effect of NLRP3 stimulation and inhibition on tumor cell viability was also confirmed by activating NLRP3 by LPS/ATP and suppressing NLRP3 using glibenclamide. Similar to LPS/Nigericin, after LPS/ATP treatment the cell death increased as compared to untreated tumors whereas glibenclamide decreased the rate of LPS/ATP mediated cell death (Supplementary Figure 4).

Next, LDH levels were analyzed to determine plasma membrane integrity in cells after modulation of NLRP3 activity. Nigericin triggered the highest level of $\mathrm{LDH}$ release in all cell lines compared to untreated controls ( $\mathrm{p}<0.001$ ). Nigericin caused the highest release of LDH in PC3 $(47.87 \%$ increase, Figure 2D) and U138MG (36.64\% increase, Supplementary Figure 3D) compared to investigated cell lines. Also, the release of LDH was lower in MCF7 (25.08\% increase, Supplementary Figure 3F) and A549 cells (13.22\% increase, Supplementary Figure $\mathbf{3 H}$ ) compared to investigated cell lines. Although the LDH release in SH-SY5Y cells was 3.50\% greater than in untreated control (Figure $\mathbf{2 H}$ ), it was still the lowest compared to all cell lines (Figures 2D, F, H; Supplementary Figures 3D, F, H, Supplementary Table 3). Additionally, LPS/ Nigericin significantly increased LDH release in all cell lines investigated ( $\mathrm{p}<0.001$ ). However, the impact of LPS/Nigericin on $\mathrm{LDH}$ release was lower than Nigericin treatment alone in all cell lines.

To determine whether NLRP3 could affect the tumor proliferation, cells were treated with VX765, LPS, Nigericin and LPS/Nigericin before real-time monitoring of cell division using the xCELLigence biosensor for $24 \mathrm{~h}$. VX765 and LPS did not affect cell proliferation compared to untreated controls in all investigated cells. In contrast, Nigericin and LPS/Nigericin substantially inhibited cell proliferation in all tumor cell lines. The effect of NLRP3 modulation of cell proliferation in PC3 cells, where inflammasome expression was the highest, and in SH-SY5Y, with the lowest NLRP3 expressions upon after Nigericin and LPS/Nigericin treatment, are shown in Figures 2E, G. Additionally, the effect of NLRP3 modulation on cell proliferation in U138MG, MCF7 and A549 cells is shown in Supplementary Figures 3E, G, I. Interestingly, the proliferation pattern of fibroblasts did not change after treatment with Nigericin or LPS/Nigericin and it remained similar to that in untreated controls (Figure 2I). It appears that the effect of NLRP3 activation on tumor growth depends on the stimulus. 
A

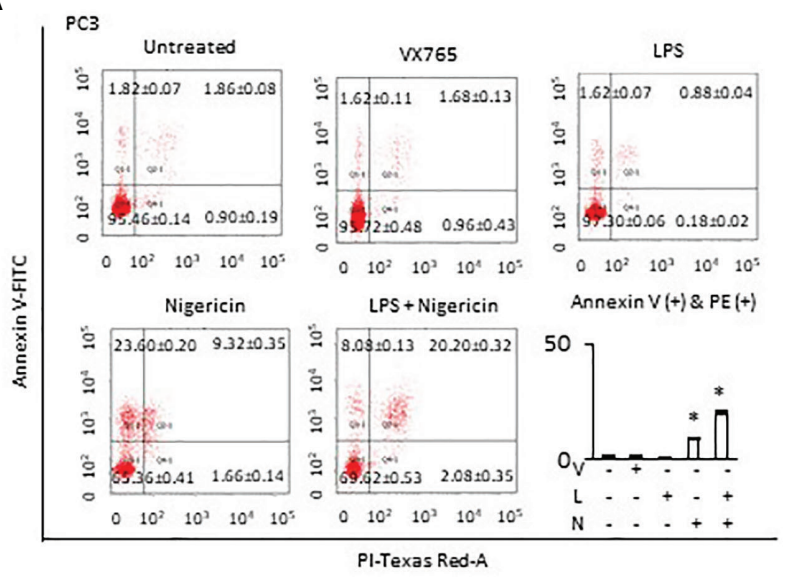

B

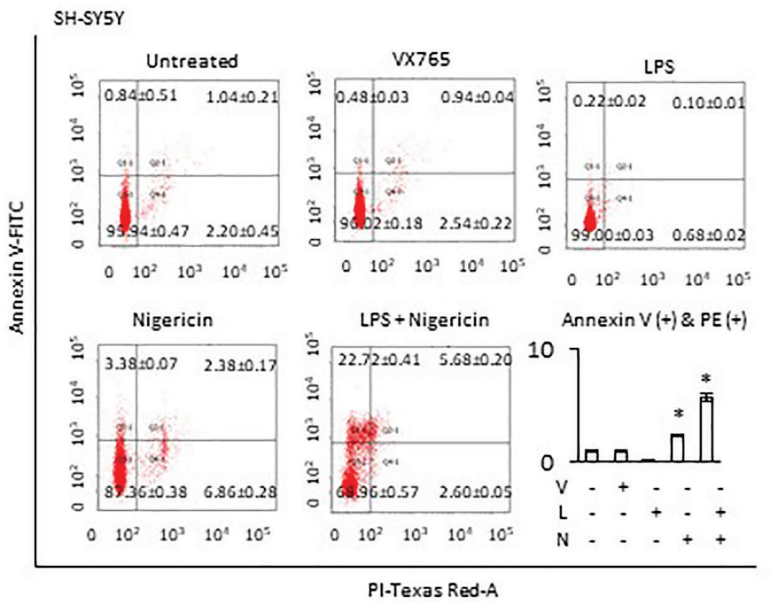

C

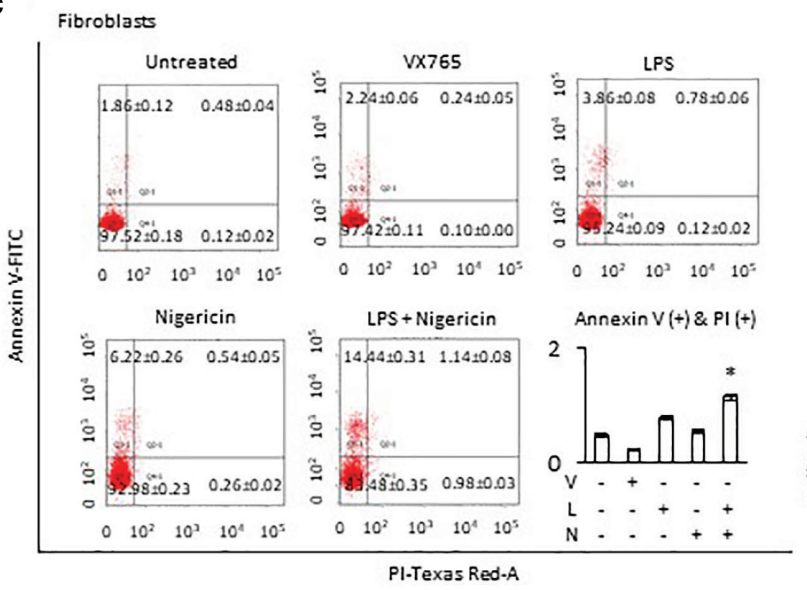

D

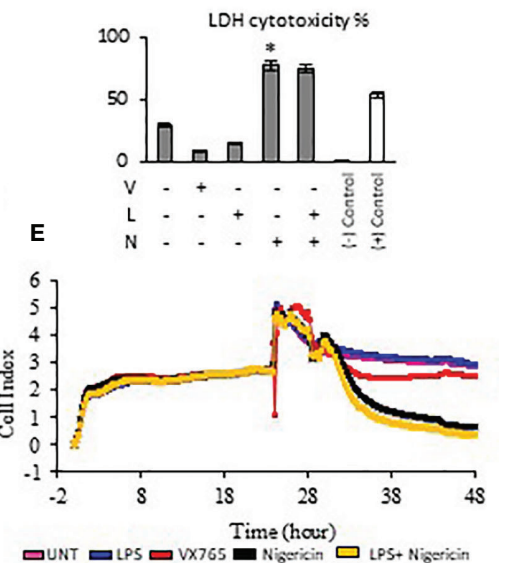

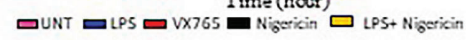

$\mathbf{F}$

LDH cytotoxicity \%s
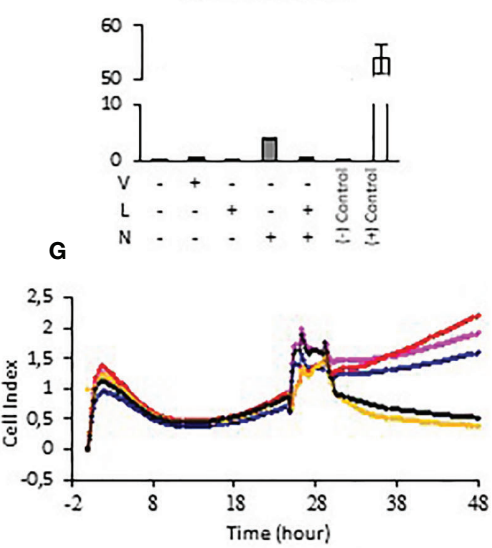

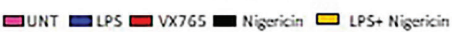

H

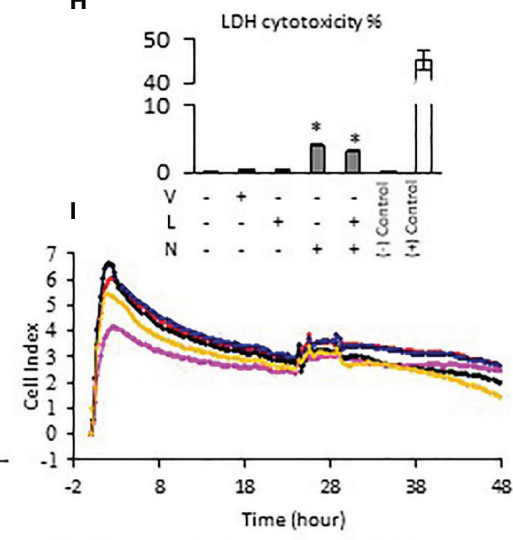

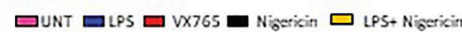

FIGURE 2 | Effect of NLRP3 inhibition and stimulation on cell viability and proliferation of PC3, SH-SY5Y cells, and Fibroblasts. Nigericin (20 $\mu \mathrm{M})$, treatment for $24 \mathrm{~h}$ with and without $3 \mathrm{~h}$ pre-incubation with LPS $(1 \mu \mathrm{g} / \mathrm{mll})$, was used to activate the NLRP3 inflammasome. To inhibit Caspase 1, cells were treated with VX765 (20 $\mu$ M). (A-C) Expression of Annexin $\mathbf{V}(\mathbf{D}, \mathbf{F}, \mathbf{H})$ LDH cytotoxicity assay and (E, G, I) a real-time cell proliferation assay. U, Untreated; V, VX765; L, LPS; N, Nigericin; LN, LPS/Nigericin. ${ }^{*} \mathrm{p}<0.05, \mathrm{n}=3$. 
While LPS did not significantly affect the cell viability and proliferation, adding the second stimulus, Nigericin, induced cell death.

The tumorsphere formation assay was used to assess stem cell-like characteristics of cancer cell lines after modulation of the NLRP3 inflammasome. Similar to our findings of cell viability and proliferation, VX765 and LPS did not affect the tumorsphere size (Figure 3). In contrast, while Nigericin and LPS/Nigericin consistently decreased cell proliferation and increased cell death in tumor cell monolayers, they had different effects on tumorsphere size across the cell lines investigated. In MCF7 and SH-SY5Y cells, Nigericin and LPS/Nigericin treatments significantly decreased sphere sizes compared to untreated controls $(\mathrm{P}<0.001$, Figure 3). However, in PC3 and U138MG cells, Nigericin did not affect tumor size, while LPS/Nigericin significantly increased the sphere sizes compared to untreated controls $(\mathrm{P}<0.001$, Figure 3). Nigericin and LPS/Nigericin did not affect the sphere size in A549 cells compared to untreated controls (Figure 3). Collectively, our data suggest that high activation of NLRP3 by LPS/Nigericin results in the growth of tumorsphere whereas low NLRP3 activation by LPS/Nigericin results in a reduction of tumorsphere size.

\section{VX765 Increases the Release of CCL24, While Nigericin Increases MIF Production}

Cell culture medium was collected $24 \mathrm{~h}$ after treatment with VX765, LPS, Nigericin and LPS/Nigericin and used to analyze cytokine release patterns. Additionally, to determine whether these cytokine patterns differ from those induced during apoptosis, the culture medium was collected from cells treated with Camptothecin (6 $\mu \mathrm{M}$ for $24 \mathrm{~h}$ ), an apoptosis inducing agent. VX765, LPS, Nigericin and LPS/Nigericin significantly modified cytokine production by tumor cells and fibroblasts (Figure 4 and Table 1). CCL24 release was commonly identified in A549, MCF7 and fibroblasts treated with VX765 compared to untreated controls (Supplementary Tables 4A, B, F). Interestingly, the effect of VX765 treatment differed in neuronal tumors where elevated levels of CCL11 and CCL26 were found in SH-SY5Y compared to untreated controls (Supplementary Table 4D), and no changes in cytokine levels were detected in VX765 treated U138MG cells compared to controls (Supplementary Table 4E). When NLRP3 was activated, it appears that LPS and Nigericin upregulated different cytokines in tumor cells (Figure 4, Table 1). While LPS increased the levels of multiple cytokines, Nigericin uniquely increased MIF levels in A549, PC3, SH-SY5Y, U138MG, and fibroblasts compared to untreated controls (Supplementary Tables 4A, C-F). Interestingly, a statistically significant increase in MIF levels was only found in SH-SY5Y cells when treated with LPS/ Nigericin compared Camptothecin treated controls $(p=0.008$, Supplementary Table 4D). Although not statistically significant, MIF expression was higher in A549, U138MG and fibroblasts treated with LPS/Nigericin compared to Camptothecin treated controls (Supplementary Tables 4A, E, and F). The exception was MCF7 cells, where Nigericin stimulated CXCL8 and CCL13 compared to untreated and Camptothecin treated controls without affecting MIF levels (Supplementary Table 4B).

Although the cytokine pattern did not change in cells treated with Caspase 1 inhibitor compared to untreated controls, the concentration of cytokines secreted differed significantly between cells treated with VX765 and LPS/Nigericin. Treatment with VX765 significantly increased the level of cytokines mediating angiogenesis (Table 2) compared to LPS/Nigericin in A549 and SH-SY5Y cells. In PC3 cells, although VX765 did not affect the release of most cytokines, CCL24 secretion was significantly higher compared to the LPS/Nigericin treated group ( $\mathrm{p}<0.05)$. In contrast, the level of angiogenic cytokines (Table 2) was significantly lower in VX765 treated cells than LPS/Nigericin treated PC3 cells. Similarly, the level of these angiogenic cytokines (Table 2) in VX765 treated MCF7 and U138MG was lower than LPS/Nigericin treated cells. Interestingly, VX765 did not affect the release of cytokines in fibroblasts. Cytokines significantly altered by VX765 compare to LPS/Nigericin treated cells are summarized in Table 2 (Figure 4, Supplementary Tables 4A-F).

We found that, when the NLRP3 inflammasome is activated, two groups of tumor cells could be identified based on $\mathrm{LDH}$ release and IL1 $\beta$ secretion. IL1 $\beta$ secretion was highest in PC3 cells and it was one of the lowest in A549 cells. Additionally, in contrast to other tumor types, A549 the levels of multiple secreted cytokines were decreased after LPS/Nigericin treatment compared to untreated controls (Table 1). Therefore, we sought to determine the structural differences between these two cell lines after inflammasome modulation using TEM. Human fibroblast cells were used as a normal cell control. Interestingly, VX765 did not affect the amount or shape of the ER in PC3 cells compared to controls, suggesting a lack of functional activity of this organelle (Figures 5A, D). In contrast, VX765 increased the granulation of ER in A549 cells, which could indicate that the organelle is functionally active (52) (Figures 5B, E). In fibroblasts, VX765 increased the granulation of ER. In addition, VX756 caused the ER cisternae to become expanded, branched, and irregular in shape which could indicate the over synthesis of proteins (53) (Figures 5C, F). The structures of the Golgi apparatus were similar in PC3, A549, and fibroblast cells with developed cistern stacks after VX765 treatment suggesting a considerable increase in protein export (54).

Inflammasome activation caused similar ultrastructural changes in ER in PC3, A549 and human fibroblasts cells. After the inflammasome priming with LPS, ER cisternae were expanded in PC3, A549 and human fibroblast cells (Figures 5G-I). However, the ultrastructure of the Golgi apparatus was different between PC3 and A549 cells. Unlike in PC3 cells, the Golgi apparatus was poorly developed and weakly visualized in A549 cells, which could indicate impaired protein export (Figure $\mathbf{5 H})$. When only Nigericin or LPS/Nigericin was used, ultrastructural signs of non-apoptotic, lytic cell death were identified by TEM in both tumor cell lines. These included nuclear condensation, presence of the multiple rounds 


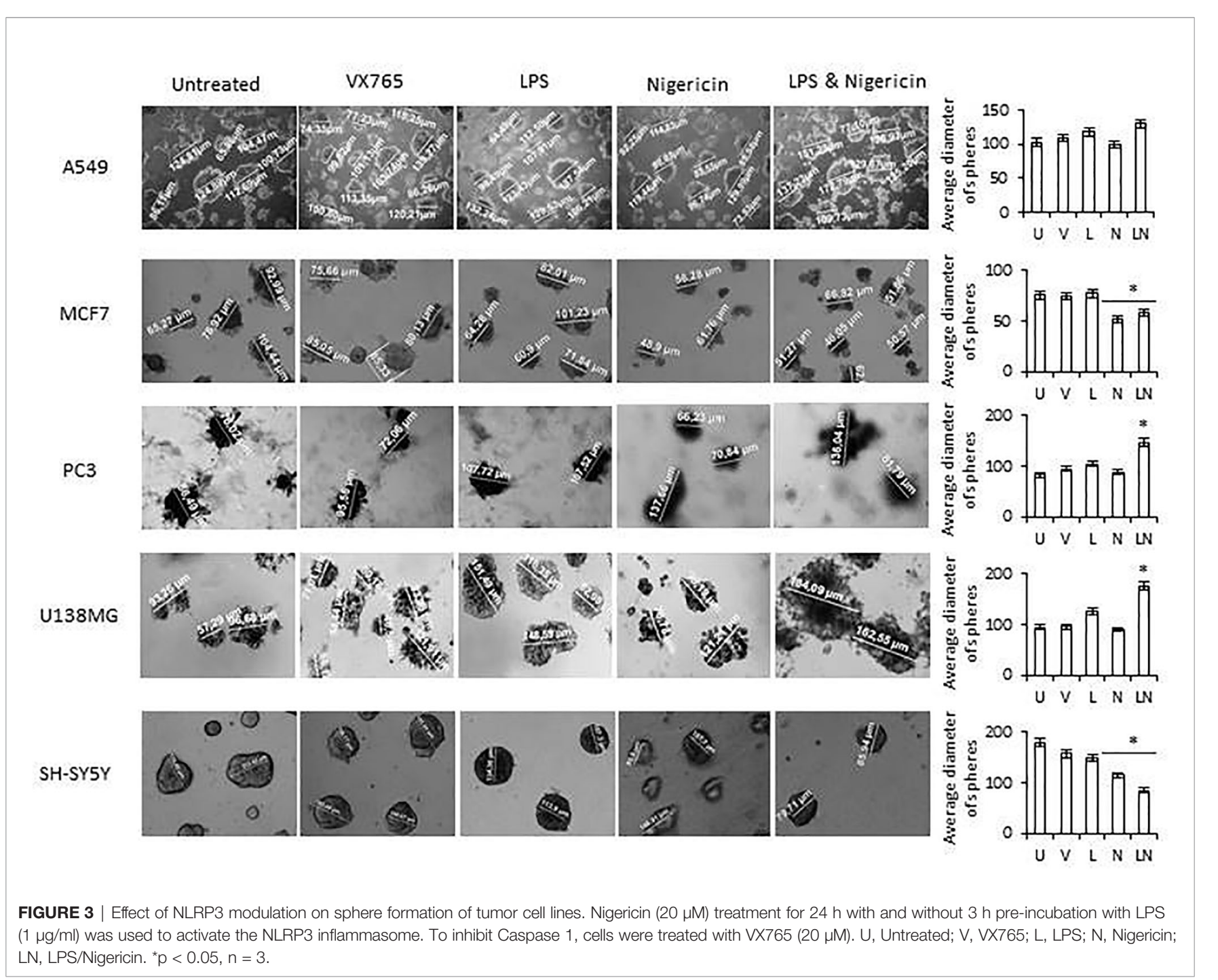

electrically transparent vacuoles in the cytoplasm, pore formation in the cell membrane, cell swelling and bursting (Figures 5J, K, M, N). In fibroblasts, LPS/Nigericin produced the ultrastructural signs of cell death similar to that in tumor cell lines. However, when used alone Nigericin had a limited effect on human fibroblasts, where few free ribosomes and many polyribosomes were identified in the cytoplasm by TEM suggesting that cells are alive rather than dead (Figure 5L).

\section{The Effect of Targeting NLRP3 Inflammasome on Mitochondria Structure and Function}

The effect of VX765, LPS, Nigericin, and LPS/Nigericin on mitochondria structure in A549, PC3, and human fibroblasts was analyzed using TEM. In untreated A549 and PC3 tumor cells, the mitochondrial cristae were poorly defined (Figures 5A, B). While VX765 did not affect the ultrastructure of mitochondria in PC3 cells (Figure 5D), in A549 cells, the outer membrane and cristae became clearer compared to untreated controls (Figure 5E). This could indicate more active ATP synthesis (55-57). In fibroblasts, after VX765 treatment, mitochondria had a condensed matrix lacking distinct cristae compared to untreated controls, suggesting increased mitochondrial activity (55) (Figures 5C, F). These data suggest that blocking Caspase 1 function, which is an NLRP3 product, does not affect the mitochondrial activity and tumor cell growth in PC3 cells. In contrast, caspase 1 inhibition induces mitochondrial activity in A549 cells and human fibroblasts.

The matrix of mitochondria was electron-dense in LPS treated PC3 cells, although the majority of cristae were condensed, more of them were clearly visible compared to untreated controls, indicating increased mitochondrial activity $(56,58)$ (Figure 5G). Similarly, in A549 cells and human fibroblasts, some cristae were clearly visible, indicating active ATP synthesis (59) (Figures 5H, I). In contrast, cristae were poorly visualized in Nigericin-treated PC3 and A549 cells, suggesting decreased mitochondrial function (60) (Figures 


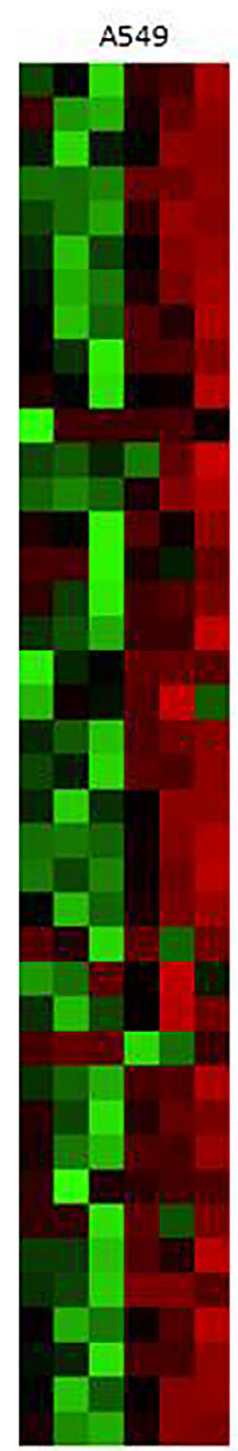

$a>-2 \equiv u$

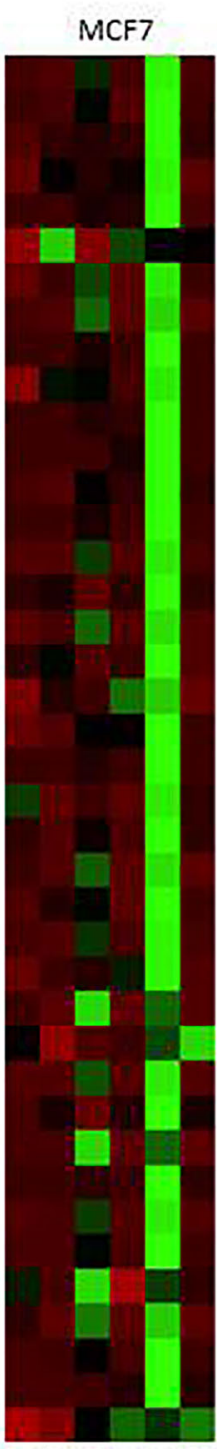

$\square>-2 \pm$

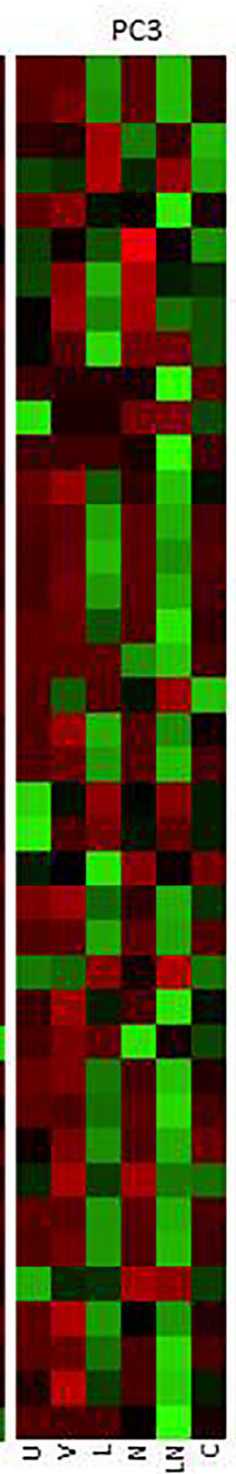

SH-SY5Y

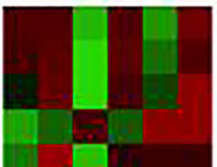

$+$
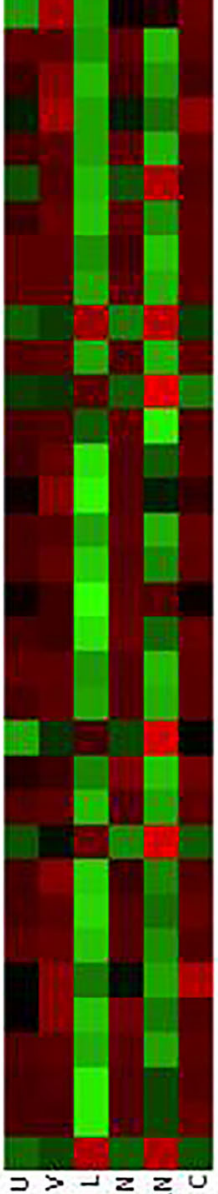

$\neg>-2 \Sigma$
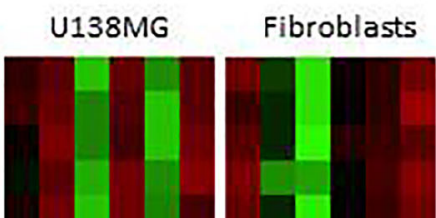

$+$
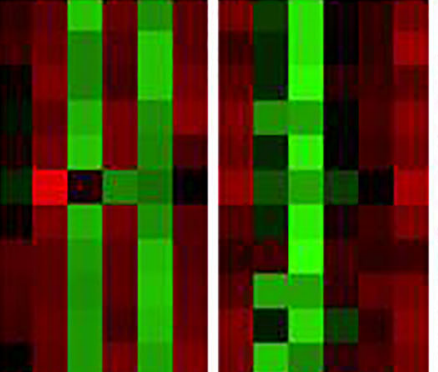

Eotaxin/CCL11

Eotaxin $2 / C C L 24$

Eotaxin-3/CO26

Fractakine? $\times 3 \mathrm{CL} 1$

GCP/CXCLE

GMCSF

Gro-a/CXCL1

Gro-b/CXCL2

1-309/CCL1

IFNg

IL16

IL2

ILA

IL6

IL8/CXCL8

IL10

IL16

IP10/CXCL10

I-TAC/CXCL11

$M C P-1 / C C L 2$

$M C P-2 / C C L 8$

MCP-3/CCL7

MCP-4/CCL13

$\mathrm{MDC} / \mathrm{CCL} 22$

MIF

MIG/CXCLS

MIP- $13 / \mathrm{CCL} 3$

MIP1b/CCL15

MIP-3a/CCL2O

MIP3-b/CCL19

MPIF-1/CCL23

SCYB16/CXCL16

SDF-1ab/CXCL12

TARC/CCL17

TERC/CCL25

TNFa

\section{U: Untreated; V:VX765; L: LPS; N: Nigericin; LN: LPS\&Nigericin; C: Camptothecin}

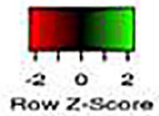

FIGURE 4 | Cytokine release patterns in A549, MCF7, PC3, SH-SY5Y, U138MG cells, and fibroblasts after NLRP3 modulation. Nigericin (20 $\mu M)$, treatment for $24 \mathrm{~h}$ with and without $3 \mathrm{~h}$ pre-incubation with LPS $(1 \mu \mathrm{g} / \mathrm{ml})$ was used to activate the NLRP3 inflammasome. To inhibit Caspase 1 , cells were treated with VX765 (20 $\mu \mathrm{M})$. Camptothecin (6 $\mathrm{MM}$, Sigma) treatment of cells for $24 \mathrm{~h}$ was used to induce apoptosis. U, Untreated; V, VX765; L, LPS; N, Nigericin; LN, LPS/Nigericin. N = 3.

5J, K). LPS/Nigericin treatment resulted in poor visualization of cristae in PC3 and A549 cell lines, indicating reduced mitochondrial function (56) (Figures 5M, N). Unlike in tumor cells, Nigericin and LPS/Nigericin produced better-defined cristae in fibroblast mitochondria compared to untreated controls, suggesting an increased mitochondrial function (56) (Figure 5L).

The modulation of NLRP3 activity on mitochondrial function was also confirmed by measuring the mitochondrial membrane potential $(\Delta \psi \mathrm{m})$ using flow cytometry analysis of JC-1 staining. The effect of NLRP3 modulation on $\Delta \psi \mathrm{m}$ in A549, MCF7 and
PC3 cell lines derived from epithelial tumors and fibroblasts is shown in Figure 6. $\Delta \psi \mathrm{m}$ changes induced by NLRP3 modulation in SH-SY5Y and U138MG cell lines, derived from neuronal tumors are shown in Supplementary Figure 5. Statistical analysis of $\Delta \psi \mathrm{m}$ changes after VX765, LPS, Nigericin and LPS\&Nigericin treatments of all cell lines is summarized in Supplementary Table 5. In PC3 and MCF7 cell lines as well as human fibroblasts, VX765 slightly decreased the right-shift of the green fluorescence indicating a reduction of the $\Delta \psi \mathrm{m}$. In contrast, there were no changes in the right-shift of the green fluorescence in VX765 treated A549 cells, suggesting a lack of 
TABLE 2 | Cytokine secretion was significantly altered by VX765 treatment compared to LPS/Nigericin treatment ( $p<0.05)$.

\begin{tabular}{|c|c|c|}
\hline \multirow[t]{2}{*}{ Cell line } & \multicolumn{2}{|c|}{ Regulation of cytokine release in VX765 treated cells compared to LPS/Nigericin treated cells } \\
\hline & Increased cytokine release & Decrease cytokine release \\
\hline A549 & $\begin{array}{l}\text { IL6, CXCL10, CXCL11, CCL1, CCL8, CCL11, CCL13, } \\
\text { CCL22,CCL24, CCL26, CCL27, TNFa }\end{array}$ & \\
\hline MCF7 & - & $\begin{array}{l}\text { IL10, IL16, IFNg, CXCL2, CXCL6, CXCL9,CCL21, CXCL10, CXCL11, CXCL13,CCL1, } \\
\text { CCL7, CCL11, CCL20, CCL23, CCL25, MIF }\end{array}$ \\
\hline PC3 & CCL24 & $\begin{array}{l}\text { IL2, IL4, IL6, IL10, IL16, IFNg, CXCL9, CXCL12, CXCL13, CCL7, CCL8, CCL11, CCL15, } \\
\text { CCL19, CCL21, CCL22, CCL23, CCL25, TNFa }\end{array}$ \\
\hline SH-SY5Y & $\begin{array}{l}\text { IL4, IFNg, CXCL1, CX3CL1, CXCL8, CXCL11, CXCL16, } \\
\text { CCL5, CCL7, CCL13, GMCSF }\end{array}$ & - \\
\hline U138 & - & $\begin{array}{l}\text { IL2, IL4, IL6, IL16, CXCL2, CXCL3, CXCL6, CXCL9, CXCL10, CCL1, CCL7, CCL17, } \\
\text { CCL20, CCL25, CCL26, CCL27, GMGSF, IFNg, MIF, TNFa }\end{array}$ \\
\hline
\end{tabular}

${ }^{*}$ Cytokines denoted in bold induce angiogenesis (39-51).

effect on $\Delta \psi \mathrm{m}$. (Figure 6, Supplementary Table 5). Interestingly, in neuronal tumors, U138MG and SH-SY5Y, VX765 increased the right-shift of the green fluorescence indicating an increased $\Delta \psi \mathrm{m}$, compared to untreated controls (Supplementary Figure 5, Supplementary Table 5). LPS slightly decreased the $\Delta \psi \mathrm{m}$ in all tumor cells; however, in human fibroblasts, LPS increased $\Delta \psi \mathrm{m}$ compared to untreated controls (Figure 6, Supplementary Table 5). Nigericin and LPS/Nigericin treatment increased $\Delta \psi \mathrm{m}$ in all tumor cells compared to untreated cells (Figure 6, Supplementary Figure 5, Supplementary Table 5). In contrast, Nigericin and LPS/ Nigericin treatment decreased $\Delta \psi \mathrm{m}$ in human fibroblasts compared to untreated controls (Figure 6, Supplementary Table 5). These data indicate that independent of the first stimulus, the second stimulus increases the mitochondria membrane potential and triggers mitochondrial dysregulation, which could cause tumor cell death. Interestingly, NLRP3 activation-induced cell death of fibroblasts requires both the first and second stimuli.

\section{Suppressing Caspase 1 Activity with VX765 Stimulates Angiogenesis}

Tumor growth is supported by neovasculogenesis, which is regulated by cytokines released from tumors (61). Therefore, we sought to determine whether modulation of NLRP3 activity could induce endothelial cell tube formation in vitro, indicating activation of angiogenesis (62). Supernatants of tumors were collected at $24 \mathrm{~h}$ and used for the HUVEC tube formation assay. HUVECs were maintained in a culture medium from each tumor cell line after NLRP3 modulation. In addition, the level of proangiogenic VEGF and Matrix metalloproteinases (MMPs) in tumor cells conditioned culture medium was analyzed. We found that in all tumor cell lines, the level of VEGF and MMP13 in culture medium was significantly higher after treatment with VX765 compared to Nigericin and LPS/Nigericin and untreated controls $(\mathrm{p}<0.05$ ) (Figure 7, Supplementary Figures 6, 7, Supplementary Tables 6, 7). Additionally, VX765 increased the release of MMP2, MMP7, and MMP10 in A549 cells, while the secretion of MMP7, MMP10, and MMP12 was increased only in PC3 cells (Figure 7, Supplementary Figure 6, Supplementary Tables 6 and 7). The level of several MMPs, including MMP2,
MMP3, MMP7, MMP10, and MMP12 was significantly higher in U138MG conditioned culture medium ( $p<0.05$ ) (Figure 7 , Supplementary Figure 7, Supplementary Tables 6 and 7). In fibroblasts, VX765 increased the release of VEGF, MMP2, MMP3, MMP10, and MMP12 compared to untreated controls, while MMP3 secretion was below the detection level (Supplementary Figure 6, Supplementary Tables 6 and 7). Consistent with the VEGF and MMPs data, VX765 culture medium induced the assembly of tubular structures by the HUVECs compared to that in controls, and Nigericin or LPS/ Nigericin treated groups $(\mathrm{p}<0.05)$ (Figure 7, Supplementary Figures 6 and 7, Supplementary Tables 6 and 7).

With exception of SH-SY5Y cells, the release of VEGF was significantly decreased in Nigericin and LPS/Nigericin treated conditioned culture medium from all tumor types (A549, MCF7, PC3, and U138MG) and fibroblasts compared to VX765 and untreated controls. Accordingly, HUVECs maintained in medium conditioned by cells treated with Nigericin and LPS/ Nigericin formed fewer tubular structures compared to VX765 treated conditioned cell culture medium. The difference in tube formation between HUVECs treated with conditioned culture medium from each tumor cell line was not significant $(\mathrm{p}<0.05)$. The effect of conditioned culture medium from PC3 cells, expressing the highest NLRP3 level upon treatment with LPS/ Nigericin, on HUVECs tube formation is shown in Figure 7. The effect of culture medium from A549 and MCF7 cells as well as fibroblasts is shown in Supplementary Figure 6. Also, the effect of culture medium from neuronal tumor cells, U138MG and SHSY5Y, on HUVEC tube formation is shown in Supplementary Figure 7. Statistical analysis of HUVEC tube formation after incubation with conditioned culture medium from VX765, LPS, Nigericin, and LPS/Nigericin treated cell lines is shown in Supplementary Table 8.

\section{DISCUSSION}

The main product of the NLRP3 inflammasome is active caspase 1 , which is produced by cleavage of the Pro-Caspase 1 (63). Caspase 1 releases the functional IL-1 3 , which is a pleiotropic cytokine inducing fever, activating and recruiting immune cells 

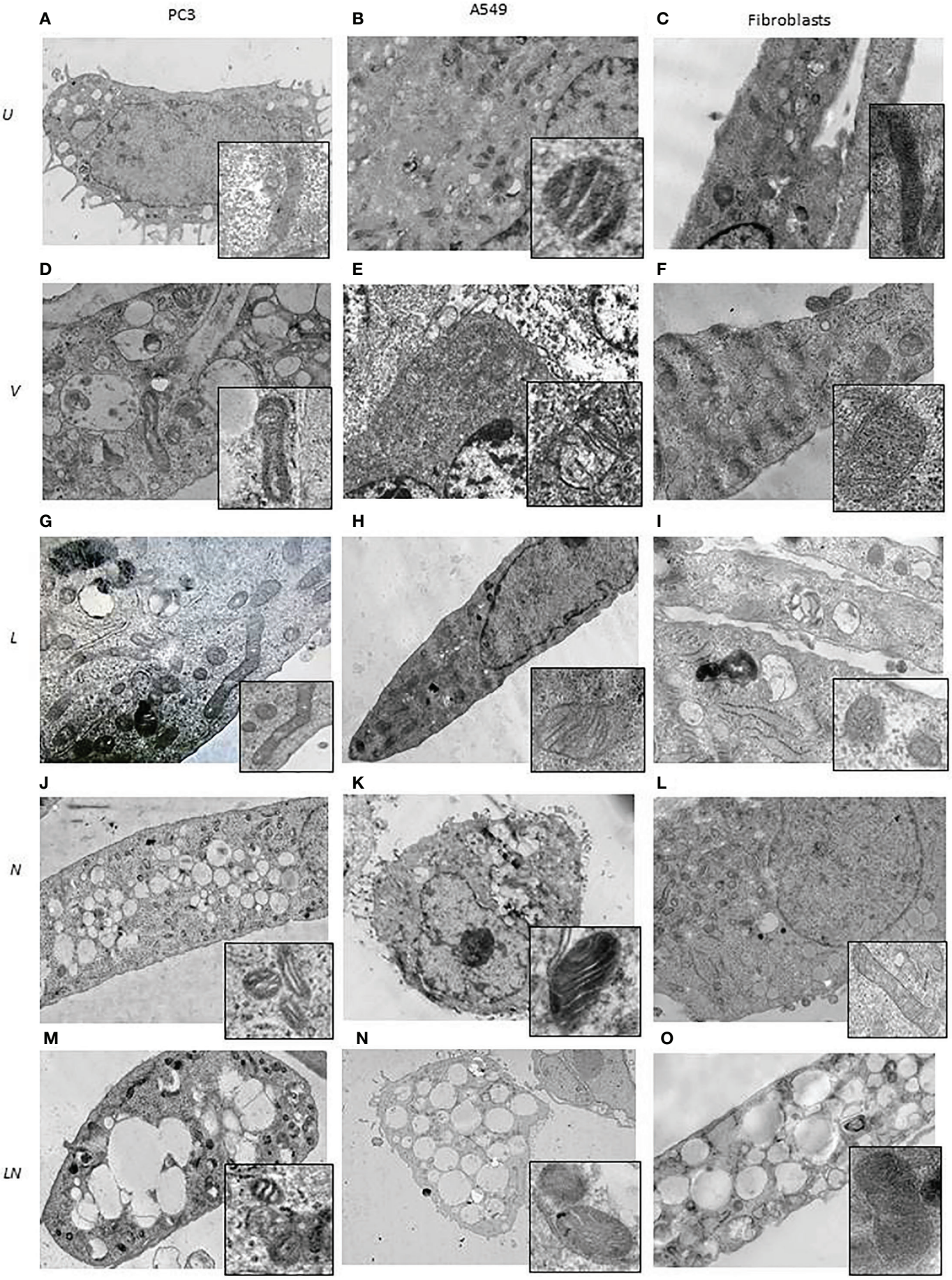

U: Untreated, $v: v x 765, L:$ LPS, $N:$ Nigericin, LN: LPS\&Nigericin

FIGURE 5 | Continued 


\begin{abstract}
FIGURE 5 | The effect of NLRP3 inflammasome modulation on cell morphology in A549, PC3, and human fibroblast cells. Nigericin (20 $\mu$ M, Invivogen) treatment for $24 \mathrm{~h}$ with and without $3 \mathrm{~h}$ pre-incubation with LPS ( $1 \mu \mathrm{g} / \mathrm{ml}$, Sigma, St. Louis, USA) was used to activate the NLRP3 inflammasome. To inhibit Caspase 1 , cells were treated with VX765 (20 $\mu \mathrm{M}$, Invivogen). (A) In untreated PC3 cells, small vacuoles were found in the cytoplasm, some of them contain electron-dense material. The cytoplasm is electron-dense containing many free ribosomes. (B) In untreated A549 cells, ER appears as narrow, elongated, rough tanks with a large number of free ribosomes. Also, oval-shaped mitochondria with clear cristae and an average electron density matrix are visualized. (C) In untreated fibroblasts, free ribosomes and polyribosomes were found in the cytoplasm. The shape of mitochondria was elongated with a condensed matrix and a large number of cristae. VX765 treatment: (D) significantly affected the ultrastructure of PC3 cells; increased numbers of large membrane-bound vacuoles were found in the cytoplasm, where some of them were merged or contained multi-vesicular aggregates. There was no visible difference in the ultrastructure of mitochondria between VX765 treated and untreated PC3 cell mitochondria. Cristae were slightly clearer compared to untreated cells. (E) In A549 cells, increased numbers of free ribosomes and granularization of ER were detected. Mitochondria were slightly enlarged and the outer membrane of mitochondria and their cristae became clear. (F) in fibroblasts, increased numbers of organelles, granularization of ER and changes to the ER structure to an expanded, branched, and irregular shape was identified. There were few free ribosomes and many polyribosomes. Additionally, the Golgi apparatus was expanded with a large number of vesicles. Mitochondria displayed a round or oval shape with condensed matrix and lacked distinct cristae. LPS treatment: (G) In PC3 cells, an increased number of organelles was identified. ER cisternae were expanded. The Golgi apparatus developed cistern stacks. Functional activity of mitochondria was increased: the ultrastructure of mitochondria was changed: the length of the mitochondria was considerably elongated, the matrix became electron-dense, and some of the cristae were visible. (H) In A549 cells, after LPS treatment; ER cisternae were expanded, had an irregular shape and formed a network. (I) In fibroblasts, similar to the effect of VX765; after LPS treatment protein synthesis and export were considerably increased; where ER cisternae were greatly expanded which had an irregular shape and formed a network. Additionally, the Golgi apparatus was visualized with well-developed cisternae stacks and a large number of vesicles in the cytoplasm. These vesicles were also detected near the cytoplasmic membrane of the cells; some of them were involved in exocytosis. After Nigericin or combined LPS and Nigericin treatments: (J, M, K, N). PC3 and A549 cells showed signs of lytic cell death such as nuclear condensation, numerous round transparent vacuoles in the cytoplasm, pore formation in the cell membrane, cell swelling and bursting. The morphology of the mitochondria was significantly changed as compared to control where: the shape of the organelle was toroidal; the matrix became electron-dense with few elongated cristae or, the absence of cristae. (L). Different from the tumor cell lines, in Fibroblasts, Nigericin treatment did not cause death cell morphology. Nigericin affected the nucleus structure where the integrity of the karyolemma appeared slightly destroyed. Mitochondria were round or oval, with clear cristae. The cytoplasm contained few free ribosomes and many polyribosomes. (0). After combined LPS and Nigericin treatment fibroblasts demonstrated a lytic cell death morphology including nuclear condensation, large electron-transparent vacuoles and pore formation in the cell membrane, cell swelling and bursting.
\end{abstract}

into the inflamed tissue (64). In this study, we investigated the effect of an inducer of the NLRP3 inflammasome, Nigericin and an inhibitor of Caspase 1, VX765, on tumor progression in a panel of cancer cells, including A549 (lung cancer), MCF7 (breast cancer), PC3 (prostate cancer), SH-SY5Y (neuroblastoma) and U138MG (glioblastoma) cell lines. We also used fibroblasts as a non-malignant cell control.

\section{The Effect of NLRP3 Activation With Nigericin}

NLRP3 complex formation requires two stimuli: priming (LPS) and activation (Nigericin) (37). LPS binds to pattern recognition receptors (PRRs) leading to the nuclear translocation of nuclear factor- $\kappa \mathrm{B}(\mathrm{NF}-\kappa \mathrm{B})$ and increased transcription of $I L 1-\beta$ and $I L-18$ (65). However, a second stimulus is required to initiate the functional inflammasome formation (66). Therefore, we analyzed the effect of LPS and Nigericin on NLRP3 activation individually and in combination. Interestingly, our results revealed that priming of tumor cells with LPS increased the release of IL1- $\beta$ and IL-18 without the requirement for the second stimulus (Nigericin). In contrast, priming of fibroblasts with LPS alone failed to release IL1- $\beta$ and IL-18 without treatment with Nigericin as a second stimulus. In addition to potassium efflux, NLRP3 can be activated by various stimuli, such as ATP, toxins, reactive oxygen species (ROS), hypoxia, and mitochondrial dysfunction $(67,68)$. Therefore, our results suggest that LPS could activate NLRP3 and cause the release of IL1- $\beta$ and IL-18 without a second stimulus provided by Nigericin. In tumor cells there could be other second stimuli already present, negating the requirement for Nigericin. Such stimuli could include oncogene-induced ROS (69).

Nigericin without LPS priming also activated NLRP3 evidenced by increased IL1- $\beta$ and IL-18 expression. It was recently shown that TLRs, FAS-associated death domain protein and IL-1R ligands can act as the NLRP3 priming stimuli $(37,70,71)$. Tarassishin and colleagues demonstrated that, in glioblastoma cells, the priming signal can be provided by IL-1 which can be produced by tumor cells in large quantities (72). Interestingly, after combined LPS/Nigericin treatment, tumor cells demonstrated two distinct patterns of NLRP3 inflammasome activation. While the release of IL-1 $\beta$ and IL-18 was higher in PC3 and U138MG cells compared to controls, these cytokines levels were lower in A549, MCF7 and SH-SY5Y cells. Microenvironment (73), redox balance $(74,75)$ and osmolarity (76) of cells may influence the release of IL- $1 \beta$ from different tumor types (77). However, little is known about the mechanism of IL-1 $\beta$ release upon LPS/Nigericin treatment. Our data suggest that NLRP3 activation through LPS/Nigericin treatment leads to a high level of IL-1 $\beta$ and IL-18 secretion in prostate cancer and glioblastoma cell lines compared to cell lines derived from lung cancer, breast cancer, and neuroblastoma.

Nigericin decreased cell viability and proliferation in all tumor cell lines investigated, both as monolayers and in sphere culture. Nigericin increases the efflux of $\mathrm{K}^{+}$and increases the intracellular $\mathrm{Ca}^{2+}$ concentration (78). According to Katsnelson and colleagues, this rapid decrease in cytosolic $\mathrm{K}^{+}$is a sufficient stimulus for initiation of the NLRP3 inflammasome cascade, independent of cytosolic $\mathrm{Ca}^{2+}$ levels (78). However, high intracellular $\mathrm{Ca}^{2+}$ can trigger apoptotic and non-apoptotic programmed cell death via caspase cascades (79). In our study, Nigericin and LPS/Nigericin increased the released LDH in tumor cells compared to controls, suggesting induction of nonapoptotic cell death. Although we suggest non-apoptotic cell death, we could not exclude pyroptosis, an inflammatory programmed cell death (80). Pyroptosis is characterized by rapidly formed membrane pores, membrane rupture, cell 


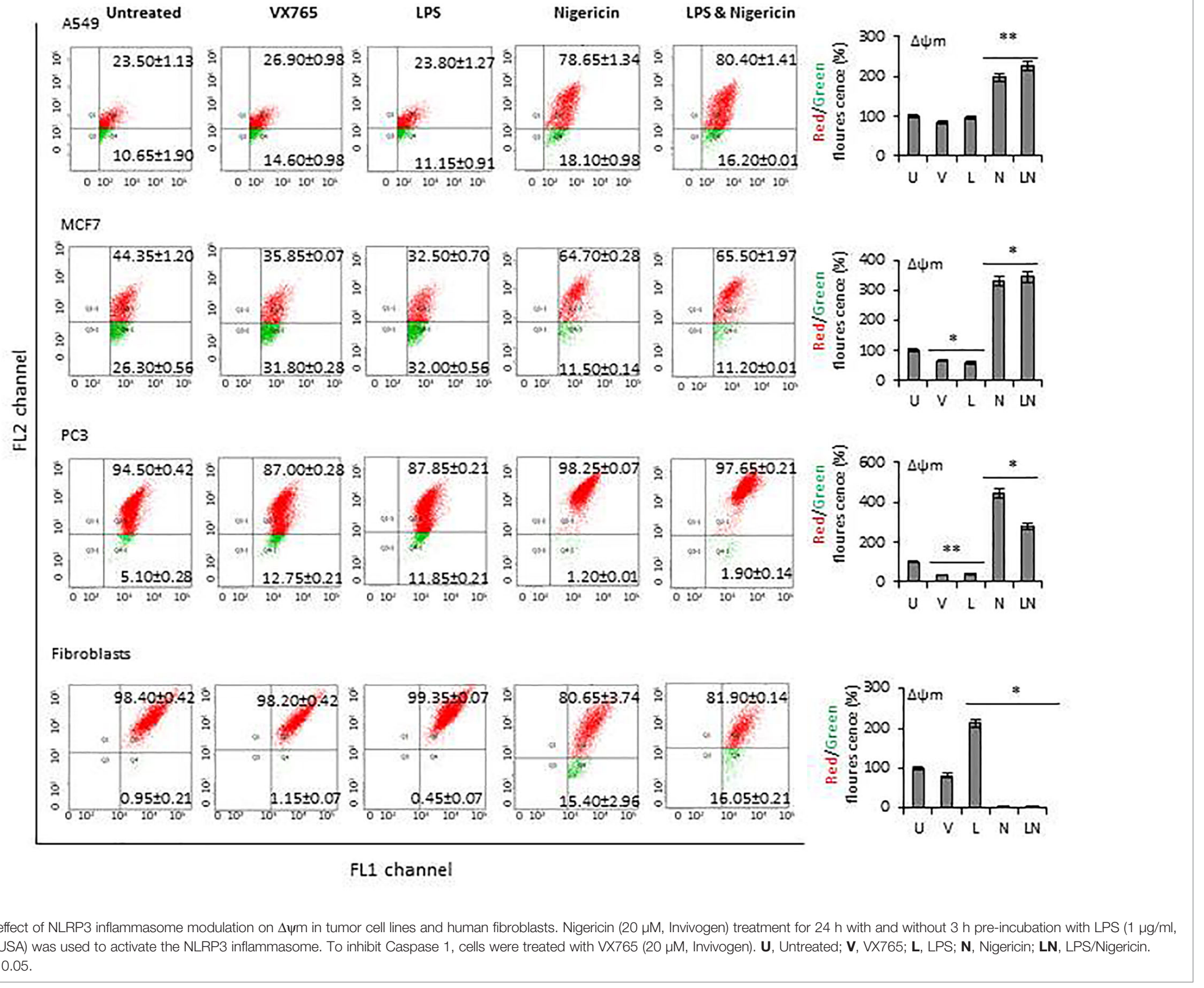


A

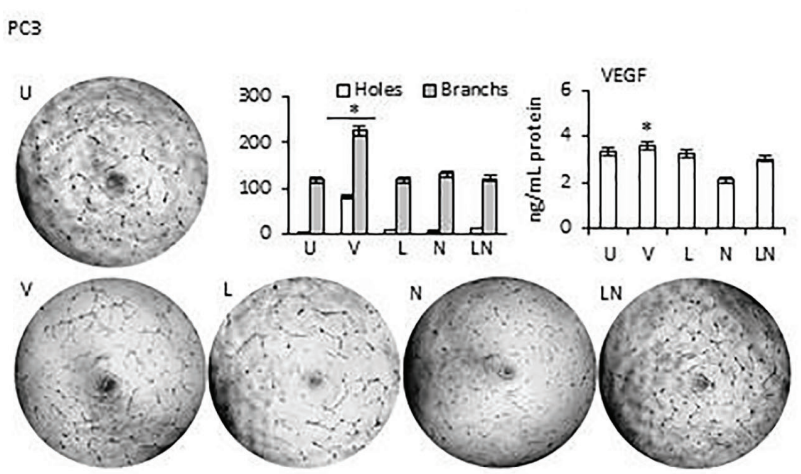

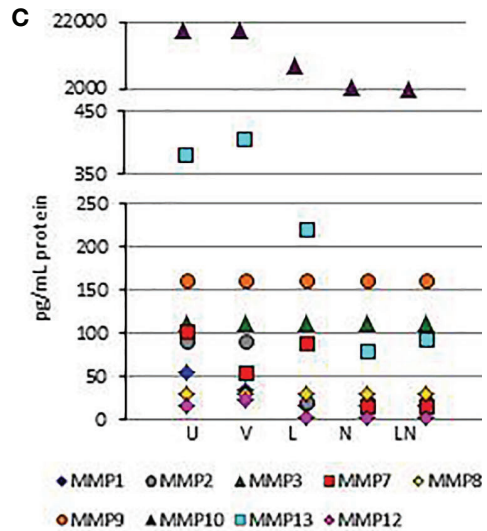

FIGURE 7 | The effects of releasing cytokines from PC3 cells after inhibition or stimulation of the NLRP3 inflammasome on the HUVEC tube formation. Nigericin ( $20 \mu \mathrm{M})$ treatment for $24 \mathrm{~h}$ with and without $3 \mathrm{~h}$ pre-incubation with LPS (1 $\mathrm{mg} / \mathrm{ml})$ was used to activate the NLRP3 inflammasome. To inhibit Caspase 1 , cells were treated with VX765 (20 $\mu M)$. U, Untreated; V, VX765; L, LPS; N, Nigericin; LN, LPS/Nigericin. * $\mathrm{p}<0.05, n=3$.

swelling and release of intracellular content into the extracellular space, including cytosolic proteins such as LDH (81). Opened membrane pores in pyroptotic cells permit both, Annexin $\mathrm{V}$ and impermanent dyes such as Propidium Iodide (PI), to enter the cell and localize in the inner membrane [39]. Therefore, in contrast to apoptosis, pyroptotic cells appear positive for both, Annexin V and PI. Supporting pyroptosis as the mechanism of cell death we identified that Nigericin and LPS/Nigericin treated tumor cells, were Annexin V and PI positive.

Additionally, Nigericin increased $\Delta \psi \mathrm{m}$ in all tumor cell lines investigated in this study. The apoptotic effect of elevated $\Delta \psi \mathrm{m}$ was demonstrated by Vander Heiden MG and colleagues (82); however, Heerdt and colleagues also showed that Nigericin induced $\Delta \psi \mathrm{m}$ is not associated with increased mitochondria-associated cytochromec release (83). Additionally, disrupted $\Delta \psi \mathrm{m}$ and reactive oxygen species were linked to pyroptosis in macrophages (84). Further support that pyroptosis was the mechanism of cell death, we found signs of oncolytic cell death in cells treated with Nigericin and LPS/ Nigericin in TEM. It appears that pyroptosis in this context is a unique form of cell death induced in tumor cells as we found no decrease in cell viability and proliferation of non-tumor cells, fibroblasts. Moreover, the effect of Nigericin on mitochondria was also different in tumors compared with fibroblasts, where the reduction of $\Delta \psi \mathrm{m}$ in fibroblasts and mitochondrial damage was negligible. These data suggest that tumor cells are more susceptible to Nigericin induced pyroptosis compared to fibroblasts.

Interestingly, while combined LPS/Nigericin treatment reduced cell proliferation in all tumor cell line monolayers, its effect on tumorsphere formation was less uniform. While combined LPS/ Nigericin treatment reduced sphere formation in MCF7 and SHSY5Y cell lines, which also had the lowest release of IL-1 $\beta$, in PC3 and U138MG cells, where the release of IL-1 $\beta$ was the highest, treatment increased the size of tumorspheres. It was previously demonstrated that IL-1 $\beta$ may promote tumor growth and invasion through activation of cancer stem cell self-renewal (85). Although monolayers are useful tools for functional tests, cell-cell and cell- extracellular environment interactions which are responsible for cell differentiation, proliferation, vitality, responsiveness to stimuli and drug metabolism cannot be represented in monolayers as they would be in the tumor mass (86). Due to disturbances in interactions with the microenvironment, tumor cell lines growing adherently can lose their polarity. It results in changes in the response of those cells to various cellular signaling events which may include to stimulation of the NLRP3 inflammasome. On the other hand, spheres more likely mimic the physical and biochemical features of a solid tumor mass due to the proper cell-cell and cellenvironment interactions (87). In sphere models, cell proliferation depends on many factors such as the location of cells, presence of initiating cancer stem cells and the level of hypoxia (86). In our study, after LPS/Nigericin treatment, the growth pattern of PC3 and U138MG cells were different in monolayer and spheres. It may be caused by pathophysiological differences between tumorspheres due to the level of inflammasome induced hypoxia which can trigger cancer stem cell self-renewal in U138MG and PC3 spheres (88). Studies demonstrated that IL-1 $\beta$ induced hypoxia in glioblastoma and modulates the tumor progression by interacting directly with the tumor cells (89). Our data suggest that the effect of Nigericin on tumor survival will depend on the balance between pyroptosis and survival associated with IL-1 $\beta$ release. Interestingly, pyroptosis could disrupt cell integrity and release the intracellular content including IL-1 $\beta$ (90). Therefore, we suggest that pyroptosis could modify the tumor microenvironment establishing a chronic inflammatory milieu by releasing pro-inflammatory cytokines (91, 92). This pro-inflammatory tumor microenvironment could also be supported by LPS, which is the priming signal in inflammasome formation (93). LPS, as a PAMP, can activate tumor-associated macrophages and trigger the release of inflammatory cytokines including TNF $\alpha$, IFN $\gamma$, IL-2, and IL-4 (94-96). Additionally, LPS can stimulate IL-6 production by tumor fibroblasts (97). Our data demonstrated that LPS induced the secreted of IL-6, as well as 31 cytokines in fibroblasts. Interestingly, in contrast to fibroblasts, fewer cytokines had 
increased secretion; secretion of only one cytokine in A549 and MCF7 cells, 5 cytokines in SH-SY5Y cells significantly increased after LPS treatment as compared to untreated cells. In addition, LPS did not affect the cytokine secretion pattern of PC3 and U138MG cells compared to untreated control. We did not identify a common pattern of cytokine secretion changes in five different tumor cell lines induced by LPS treatment. We propose that the differences in the level of NLRP3 activation in tumor cell lines are related to variations in the inflammatory nature of the individual tumor types, where pro-inflammatory cytokines produced by the tumor could act as priming agents, instead of LPS (72, 98-100).

In contrast to LPS (where no common cytokine could be identified activated in tumor cells line), Nigericin treatment revealed MIF as the most consistently upregulated cytokine in tumor cells, except for MCF7 cells. It appears that MIF could function as positive feedback to potentiate Nigericin activation of NLRP3, as this cytokine regulates inflammasome assembly and activation (101). It also was shown that MIF is required for the NLRP3-vimentin interaction, which is essential for IL-1 $\beta$ and IL-18 secretion (101). Our data demonstrate that MIF production could be involved in NLRP3 activation in tumor cells treated with Nigericin. Interestingly, Nigericin did not induce the production of MIF in MCF7 breast cancer cells; instead, these cells produced CXCL8 and CCL13. Nigericin can mimic the P2X7 receptor and trigger the second stage of NLRP3 inflammasome activation (9). It was demonstrated that P2X7 receptors were involved in the production of CXCL8 in human bronchial epithelial cells (102). Additionally, the release of CXCL8 was observed together with IL-1 $\beta$ in cigarette smokerassociated chronic obstructive pulmonary disease (COPD) patients (102). It was reported that IL-1 $\beta$ induces CXCL8 via NLRP3 (102). Therefore, we suggest that Nigericin may also lead to the production of CXCL8 in MCF7 cells. However, the role of CCL13 in the regulation of the NLRP3 inflammasome in MCF7 cells requires further investigation.

\section{The Effect of VX765 on Inflammasome Function}

VX765 is a selective inhibitor of Caspase 1, a major product and effector of the NLRP3 inflammasome (35). To confirm VX765 inhibition of Caspase 1, cells were treated with LPS/Nigericin, which activates NLRP3 to produce the active caspase. In all tumor cell lines and fibroblasts, Caspase 1 inhibition, partially attenuated the effects of LPS/Nigericin. In contrast to NLRP3 activation, Caspase 1 inhibition alone did not affect cell proliferation, death and $\mathrm{LDH}$ release. Moreover, TEM data suggest that VX765 improved the cell metabolism and mitochondrial functions in A549, PC3, and fibroblasts. Studies demonstrated that ER stress (ERS) activates the NLRP3 inflammasome and triggers mitochondrial damage. ERS increases ROS and promotes translocation of the inflammasome to the mitochondria. NLRP3 is involved in the ERS-induced cleavage of caspases including caspase-1 leading to mitochondrial damage, which is required for the production of mature IL-1 $\beta(103,104)$. During ERS, the capacity of the ER to fold proteins becomes saturated by impaired protein glycosylation or disulfide bond formation (105). According to our findings, it appears that the inhibitor function VX765 to caspase-1 results with decrease in ERS and increase in the capacity of ER to fold proteins accurately. Caspase 1 inhibition also increased production of CCL24 in epithelial tumors such as lung, breast and prostate. In contrast to epithelial tumors (A549, MCF7 and PC3), Caspase 1 inhibition triggered the release of CCL11 and CCL26 in a neuroblastoma cell line. These cytokines are ligands for CCR3, which is expressed on the surface of immune and non-immune cells and promotes cell migration and proliferation $(40,106,107)$. It was previously demonstrated that the CCL24-CCR3 interaction increases eosinophil adhesion and facilitates migration and infiltration of eosinophils (108). Infiltrated eosinophils were shown to increase tumor cell viability and their proangiogenic potential (109). CCL24 can also promote angiogenesis via the RhoB-VEGFA-VEGFR2 signaling pathway and contributing to malignancy (110). Additionally, CCL11-CCR3 interaction promotes cell migration and proliferation (110). Our data demonstrated that Caspase 1 inhibition induces the release of VEGF and MMPs from tumor cells and stimulates endothelial cell tube formation. This is further supported by our data on decreased cell death in tumor cells when Caspase 1 was inhibited. Our data is also corroborated LopezPastrana and colleagues' findings, who demonstrated that inhibition of Caspase 1 reduces pyroptosis and stimulates endothelial cell survival, which is mediated by VEGFR-2 signaling (111). Altogether our data suggest that inhibiting Caspase 1 by VX765 induces angiogenesis.

In conclusion, in this study, we, for the first time, analyzed the effect of an inducer (Nigericin) of NLRP3 and inhibitor of Caspase 1 (VX765) in a panel of different tumor types and normal fibroblast controls. We identified that the degree of inflammasome activation varies across the investigated tumor cell lines. Upon LPS/Nigericin treatment, activation of NLRP3 was the highest in prostate cancer and glioblastoma cells, whilst it was the lowest in the neuroblastoma cell line, SH-SY5Y. Additionally, for the first time, we have demonstrated that cell death caused by LPS/Nigericin treatment, produced variable effects on tumor cells, depending upon their NLRP3 activation level and cytokines released. In this study, we used only one cell line for each tumor type. Considering the heterogeneous nature of every tumor type, the efficacy of the inflammasome activation could further vary in different tumor cells derived from the same cancer type. Additionally, although inflammasome targeting is used as a therapeutic approach in many diseases, our study showed that inhibition of NLRP3 may not be the best approach for the treatment of some cancers. This is because the inhibiting of Caspase 1 activity using VX765 could stimulate the angiogenesis by releasing CCL24, CCL11, and CCL26 cytokines and protecting cell viability in some tumors. Therefore, targeting inflammasomes for cancer treatment will require prior testing of the effect of inflammasome reactivation in that tumor. Future invivo investigations will better clarify the efficiency of inflammasome activation and Caspase-1 inhibition on tumor progression. However, according to our current findings, inflammasomes could be excellent targets for personalized cancer treatment, as 
analysis of inflammasome activation status and potential outcome of their targeting in each patient could identify a supplemental strategy to control tumor growth if appropriate biomarkers can be identified and validated.

\section{DATA AVAILABILITYSTATEMENT}

The original contributions presented in the study are included in the article/Supplementary Material; further inquiries can be directed to the corresponding author.

\section{REFERENCES}

1. Zhang H, Lu J, Lu Y, Zhou J, Wang Z, Liu H, et al. Prognostic significance and predictors of the system inflammation score in ovarian clear cell carcinoma. PLoS One (2017) 12(5):e0177520. doi: 10.1371/journal.pone.0177520

2. Li J, Yang F, Wei F, Ren X. The role of toll-like receptor 4 in tumor microenvironment. Oncotarget (2017) 8(39):66656-67. doi: 10.18632/ oncotarget.19105

3. Afonina IS, Zhong Z, Karin M, Beyaert R. Limiting inflammation-the negative regulation of NF- $\mathrm{KB}$ and the NLRP3 inflammasome. Nat Immunol (2017) 18(8):861. doi: 10.1038/ni.3772

4. He Q, Fu Y, Tian D, Yan W. The contrasting roles of inflammasomes in cancer. Am J Cancer Res (2018) 8(4):566.

5. Koido S, Homma S, Okamoto M, Namiki Y, Takakura K, Uchiyama K, et al. The combination of TLR2 and TLR4 agonists promotes the immunogenicity of dendritic cell/cancer cell fusions. Oncoimmunology (2013) 2(7):e24660. doi: 10.4161/onci.24660

6. Bauernfeind FG, Horvath G, Stutz A, Alnemri ES, MacDonald K, Speert D, et al. Cutting edge: NF-kappaB activating pattern recognition and cytokine receptors license NLRP3 inflammasome activation by regulating NLRP3 expression. J Immunol (2009) 183(2):787-91. doi: 10.4049/ jimmunol.0901363

7. Tezcan G, Martynova EV, Gilazieva ZE, McIntyre A, Rizvanov AA, Khaiboullina SF. MicroRNA Post-transcriptional Regulation of the NLRP3 Inflammasome in Immunopathologies. Front Pharmacol (2019) 10:451. doi: 10.3389/fphar.2019.00451

8. Costa A, Gupta R, Signorino G, Malara A, Cardile F, Biondo C, et al. Activation of the NLRP3 inflammasome by group B streptococci. J Immunol (2012) 188(4):1953-60. doi: 10.4049/jimmunol.1102543

9. Perregaux D, Gabel CA. Interleukin-1 beta maturation and release in response to ATP and nigericin. Evidence that potassium depletion mediated by these agents is a necessary and common feature of their activity. J Biol Chem (1994) 269(21):15195-203.

10. Petrilli V, Papin S, Dostert C, Mayor A, Martinon F, Tschopp J. Activation of the NALP3 inflammasome is triggered by low intracellular potassium concentration. Cell Death Differ (2007) 14(9):1583. doi: 10.1038/ sj.cdd.4402195

11. Okada M, Matsuzawa A, Yoshimura A, Ichijo H. The lysosome ruptureactivated TAK1-JNK pathway regulates NLRP3 inflammasome activation. J Biol Chem (2014) 289(47):32926-36. doi: 10.1074/jbc.M114.579961

12. Heid ME, Keyel PA, Kamga C, Shiva S, Watkins SC, Salter RD. Mitochondrial reactive oxygen species induces NLRP3-dependent lysosomal damage and inflammasome activation. J Immunol (2013) 191 (10):5230-8. doi: 10.4049/jimmunol.1301490

13. Gurung P, Lukens JR, Kanneganti T-D. Mitochondria: diversity in the regulation of the NLRP3 inflammasome. Trends Mol Med (2015) 21 (3):193-201. doi: 10.1016/j.molmed.2014.11.008

14. Koizumi Y, Toma C, Higa N, Nohara T, Nakasone N, Suzuki T. Inflammasome activation via intracellular NLRs triggered by bacterial infection. Cell Microbiol (2012) 14(2):149-54. doi: 10.1111/j.14625822.2011.01707.x

\section{AUTHOR CONTRIBUTIONS}

All authors listed have made a substantial, direct, and intellectual contribution to the work and approved it for publication.

\section{SUPPLEMENTARY MATERIAL}

The Supplementary Material for this article can be found online at: https://www.frontiersin.org/articles/10.3389/fimmu.2020. 607881/full\#supplementary-material

15. Franchi L, Muñoz-Planillo R, Núñez G. Sensing and reacting to microbes through the inflammasomes. Nat Immunol (2012) 13(4):325. doi: 10.1038/ ni. 2231

16. Alnemri ES, Livingston DJ, Nicholson DW, Salvesen G, Thornberry NA, Wong WW, et al. Human ICE/CED-3 protease nomenclature. Cell (1996) 87 (2):171. doi: 10.1016/S0092-8674(00)81334-3

17. Akira S, Takeda K, Kaisho T. Toll-like receptors: critical proteins linking innate and acquired immunity. Nat Immunol (2001) 2(8):675. doi: 10.1038/ 90609

18. Wang H, Luo Q, Feng X, Zhang R, Li J, Chen F. NLRP3 promotes tumor growth and metastasis in human oral squamous cell carcinoma. BMC Cancer (2018) 18(1):500. doi: 10.1186/s12885-018-4403-9

19. Feng X, Luo Q, Zhang H, Wang H, Chen W, Meng G, et al. The role of NLRP3 inflammasome in 5-fluorouracil resistance of oral squamous cell carcinoma. J Exp Clin Cancer Res (2017) 36(1):81. doi: 10.1186/s13046-017-0553-x

20. Huang C-F, Chen L, Li Y-C, Wu L, Yu G-T, Zhang W-F, et al. NLRP3 inflammasome activation promotes inflammation-induced carcinogenesis in head and neck squamous cell carcinoma. J Exp Clin Cancer Res (2017) 36 (1):116. doi: 10.1186/s13046-017-0589-y

21. Yin X-F, Zhang Q, Chen Z-Y, Wang H-F, Li X, Wang H-X, et al. NLRP3 in human glioma is correlated with increased WHO grade, and regulates cellular proliferation, apoptosis and metastasis via epithelial-mesenchymal transition and the PTEN/AKT signaling pathway. Int J Oncol (2018) 53 (3):973-86. doi: 10.3892/ijo.2018.4480

22. Kolb R, Liu G-H, Janowski AM, Sutterwala FS, Zhang W. Inflammasomes in cancer: a double-edged sword. Protein Cell (2014) 5(1):12-20. doi: 10.1007/ s13238-013-0001-4

23. Okamoto M, Liu W, Luo Y, Tanaka A, Cai X, Norris DA, et al. Constitutively active inflammasome in human melanoma cells mediating autoinflammation via caspase-1 processing and secretion of interleukin-1ß. J Biol Chem (2010) 285(9):6477-88. doi: 10.1074/jbc.M109.064907

24. Lin C, Zhang J. Inflammasomes in inflammation-induced cancer. Front Immunol (2017) 8:271. doi: 10.3389/fimmu.2017.00271

25. Karki R, Man SM, Kanneganti T-D. Inflammasomes and cancer. Cancer Immunol Res (2017) 5(2):94-9. doi: 10.1158/2326-6066.CIR-16-0269

26. Zahid A, Li B, Kombe JK, Jin T, Tao J. Pharmacological Inhibitors of the NLRP3 Inflammasome. Front Immunol (2019) 10:2538. doi: 10.3389/fimmu.2019.02538

27. Strangward P, Haley MJ, Albornoz MG, Barrington J, Shaw T, Dookie R, et al. Targeting the IL33-NLRP3 axis improves therapy for experimental cerebral malaria. Proc Natl Acad Sci (2018) 115(28):7404-9. doi: 10.1073/ pnas. 1801737115

28. Mariathasan S, Weiss DS, Newton K, McBride J, O’Rourke K, Roose-Girma $\mathrm{M}$, et al. Cryopyrin activates the inflammasome in response to toxins and ATP. Nature (2006) 440(7081):228. doi: 10.1038/nature04515

29. Locovei S, Scemes E, Qiu F, Spray DC, Dahl G. Pannexin1 is part of the pore forming unit of the P2X7 receptor death complex. FEBS Lett (2007) 581 (3):483-8. doi: 10.1016/j.febslet.2006.12.056

30. Yakisich JS, Azad N, Kaushik V, O’Doherty GA, Iyer AKV. Nigericin decreases the viability of multidrug-resistant cancer cells and lung tumorspheres and potentiates the effects of cardiac glycosides. Tumor Biol (2017) 39(3):1010428317694310. doi: 10.1177/1010428317694310 
31. Liu F, Li W, Hua S, Han Y, Xu Z, Wan D, et al. Nigericin exerts anticancer effects on human colorectal cancer cells by inhibiting $\mathrm{Wnt} / \beta$-catenin signaling pathway. Mol Cancer Ther (2018) 17(5):952-65. doi: 10.1158/ 1535-7163.MCT-17-0906

32. Xu Z, Shen J, Hua S, Wan D, Chen Q, Han Y, et al. High-throughput sequencing of circRNAs reveals novel insights into mechanisms of nigericin in pancreatic cancer. BMC Genomics (2019) 20(1):1-11. doi: 10.1186/s12864-019-6032-3

33. Fernando G, Paul F, Laura J, Alejandra AM, Gabriela M, Alberto PL. Is the $\mathrm{Wnt} / \beta$ catenin signalling pathway activated in Seminoma?: An immunohistochemical study. J Cancer Res Ther (2016) 12(2):1075. doi: 10.4103/0973-1482.147392

34. Deng C-C, Liang Y, Wu M-S, Feng F-T, Hu W-R, Chen L-Z, et al. Nigericin selectively targets cancer stem cells in nasopharyngeal carcinoma. Int $J$ Biochem Cell Biol (2013) 45(9):1997-2006. doi: 10.1016/j.biocel.2013.06.023

35. Wannamaker W, Davies R, Namchuk M, Pollard J, Ford P, Ku G, et al. (S)-1((S)-2-\{[1-(4-amino-3-chloro-phenyl)-methanoyl]-amino\}-3, 3-dimethylbutanoyl)-pyrrolidine-2-carboxylic acid ((2R,3S)-2-ethoxy-5-oxotetrahydro-furan-3-yl)-amide (VX-765), an orally available selective interleukin (IL)-converting enzyme/caspase-1 inhibitor, exhibits potent anti-inflammatory activities by inhibiting the release of IL-1 $\beta$ and IL-18. J Pharmacol Exp Ther (2007) 321(2):509-16. doi: 10.1124/jpet.106.111344

36. Maroso M, Balosso S, Ravizza T, Liu J, Bianchi M, Vezzani A. Interleukin-1 type 1 receptor/Toll-like receptor signalling in epilepsy: the importance of IL-1beta and high-mobility group box 1. J Internal Med (2011) 270(4):31926. doi: $10.1111 / j .1365-2796.2011 .02431 . x$

37. He Y, Hara H, Núñez G. Mechanism and regulation of NLRP3 inflammasome activation. Trends Biochem Sci (2016) 41(12):1012-21. doi: 10.1016/j.tibs.2016.09.002

38. Lagarkova MA, Shutova MV, Bogomazova AN, Vassina EM, Glazov EA, Zhang P, et al. Induction of pluripotency in human endothelial cells resets epigenetic profile on genome scale. Cell Cycle (2010) 9(5):937-46. doi: 10.4161/cc.9.5.10869

39. Ridiandries A, Tan JT, Bursill CA. The Role of CC-Chemokines in the Regulation of Angiogenesis. Int J Mol Sci (2016) 17(11):1856. doi: 10.3390/ ijms17111856. doi: ijms17111856

40. Park JY, Kang YW, Choi BY, Yang YC, Cho BP, Cho WG. CCL11 promotes angiogenic activity by activating the PI3K/Akt pathway in HUVECs. J Recept Signal Transduct Res (2017) 37(4):416-21. doi: 10.1080/10799893.2017.1298132

41. Owen JL, Mohamadzadeh M. Macrophages and chemokines as mediators of angiogenesis. Front Physiol (2013) 4:159. doi: 10.3389/fphys.2013.00159

42. Ribatti D. Interleukins as modulators of angiogenesis and anti-angiogenesis in tumors. Cytokine (2019) 118:3-7. doi: 10.1016/j.cyto.2018.10.022. doi: S1043-4666(18)30410-1

43. Bae J, Park D, Lee YS, Jeoung D. Interleukin-2 promotes angiogenesis by activation of Akt and increase of ROS. J Microbiol Biotechnol (2008) 18 (2):377-82.

44. Lan Q, Lai W, Zeng Y, Liu L, Li S, Jin S, et al. CCL26 Participates in the PRL3-Induced Promotion of Colorectal Cancer Invasion by Stimulating TumorAssociated Macrophage Infiltration. Mol Cancer Ther (2018) 17(1):276-89. doi: 10.1158/1535-7163.MCT-17-0507

45. Hwang J, Son KN, Kim CW, Ko J, Na DS, Kwon BS, et al. Human CC chemokine CCL23, a ligand for CCR1, induces endothelial cell migration and promotes angiogenesis. Cytokine (2005) 30(5):254-63. doi: 10.1016/ j.cyto.2005.01.018

46. Zhang M, Qiu L, Zhang Y, Xu D, Zheng JC, Jiang L. CXCL12 enhances angiogenesis through CXCR7 activation in human umbilical vein endothelial cells. Sci Rep (2017) 7(1):8289. doi: 10.1038/s41598-017-08840-y

47. Zhao J, Chen L, Shu B, Tang J, Zhang L, Xie J, et al. Granulocyte/macrophage colony-stimulating factor influences angiogenesis by regulating the coordinated expression of VEGF and the Ang/Tie system. PLoS One (2014) 9(3):e92691. doi: 10.1371/journal.pone.0092691

48. Zhang J, Yang W, Luo B, Hu B, Maheshwari A, Fallon MB. The role of CX(3) CL1/CX(3)CR1 in pulmonary angiogenesis and intravascular monocyte accumulation in rat experimental hepatopulmonary syndrome. $J$ Hepatol (2012) 57(4):752-8. doi: 10.1016/j.jhep.2012.05.014

49. Hwang J, Kim CW, Son KN, Han KY, Lee KH, Kleinman HK, et al. Angiogenic activity of human CC chemokine CCL15 in vitro and in vivo. FEBS Lett (2004) 570(1-3):47-51. doi: 10.1016/j.febslet.2004.06.023
50. Kim MJ, Sun HJ, Song YS, Yoo SK, Kim YA, Seo JS, et al. CXCL16 positively correlated with M2-macrophage infiltration, enhanced angiogenesis, and poor prognosis in thyroid cancer. Sci Rep (2019) 9(1):13288. doi: 10.1038/ s41598-019-49613-Z

51. Keeley EC, Mehrad B, Strieter RM. CXC chemokines in cancer angiogenesis and metastases. Adv Cancer Res (2010) 106:91-111. doi: 10.1016/S0065230X(10)06003-3

52. Voeltz GK, Rolls MM, Rapoport TA. Structural organization of the endoplasmic reticulum. EMBO Rep (2002) 3(10):944-50. doi: 10.1093/ embo-reports/kvf202

53. Palade G. Intracellular aspects of the process of protein synthesis. Science (1975) 189(4200):347-58. doi: 10.1126/science.1096303

54. Anitei M, Hoflack B. Exit from the trans-Golgi network: from molecules to mechanisms. Curr Opin Cell Biol (2011) 23(4):443-51. doi: 10.1016/ j.ceb.2011.03.013

55. Quintana-Cabrera R, Mehrotra A, Rigoni G, Soriano M. Who and how in the regulation of mitochondrial cristae shape and function. Biochem Biophys Res Commun (2018) 500(1):94-101. doi: 10.1016/j.bbrc.2017.04.088

56. Burke PJ. Mitochondria, bioenergetics and apoptosis in cancer. Trends Cancer (2017) 3(12):857-70. doi: 10.1016/j.trecan.2017.10.006

57. Ogura A, Oowada S, Kon Y, Hirayama A, Yasui H, Meike S, et al. Redox regulation in radiation-induced cytochrome $\mathrm{c}$ release from mitochondria of human lung carcinoma A549 cells. Cancer Lett (2009) 277(1):64-71. doi: 10.1016/j.canlet.2008.11.021

58. Gottlieb E, Armour S, Harris M, Thompson C. Mitochondrial membrane potential regulates matrix configuration and cytochrome $\mathrm{c}$ release during apoptosis. Cell Death Differ (2003) 10(6):709-17. doi: 10.1038/sj.cdd.4401231

59. Hackenbrock CR. Ultrastructural bases for metabolically linked mechanical activity in mitochondria: I. Reversible ultrastructural changes with change in metabolic steady state in isolated liver mitochondria. J Cell Biol (1966) 30 (2):269-97. doi: 10.1083/jcb.30.2.269

60. Strauss M, Hofhaus G, Schröder RR, Kühlbrandt W. Dimer ribbons of ATP synthase shape the inner mitochondrial membrane. EMBO J (2008) 27 (7):1154-60. doi: 10.1038/emboj.2008.35

61. Landskron G, De la Fuente M, Thuwajit P, Thuwajit C, Hermoso MA. Chronic inflammation and cytokines in the tumor microenvironment. J Immunol Res (2014) 2014:149185. doi: 10.1155/2014/149185

62. DeCicco-Skinner KL, Henry GH, Cataisson C, Tabib T, Gwilliam JC, Watson NJ, et al. Endothelial cell tube formation assay for the in vitro study of angiogenesis. J Vis $\operatorname{Exp}$ (2014) 91):e51312. doi: 10.3791/51312

63. Boucher D, Monteleone M, Coll RC, Chen KW, Ross CM, Teo JL, et al. Caspase-1 self-cleavage is an intrinsic mechanism to terminate inflammasome activity. J Exp Med (2018) 215(3):827-40. doi: 10.1084/jem.20172222

64. Caspase-1: an integral regulator of innate immunity. Semin Immunopathol (2015). 37(4):419-27. doi: 10.1007/s00281-015-0494-4

65. Rivera A, Siracusa MC, Yap GS, Gause WC. Innate cell communication kickstarts pathogen-specific immunity. Nat Immunol (2016) 17(4):356. doi: 10.1038/ni.3375

66. Jo E-K, Kim JK, Shin D-M, Sasakawa C. Molecular mechanisms regulating NLRP3 inflammasome activation. Cell Mol Immunol (2016) 13(2):148. doi: $10.1038 / \mathrm{cmi} .2015 .95$

67. Piccini A, Carta S, Tassi S, Lasiglié D, Fossati G, Rubartelli A. ATP is released by monocytes stimulated with pathogen-sensing receptor ligands and induces IL-1 $\beta$ and IL-18 secretion in an autocrine way. Proc Natl Acad Sci (2008) 105(23):8067-72. doi: 10.1073/pnas.0709684105

68. Dostert C, Pétrilli V, Van Bruggen R, Steele C, Mossman BT, Tschopp J. Innate immune activation through Nalp3 inflammasome sensing of asbestos and silica. Science (2008) 320(5876):674-7. doi: 10.1126/science.1156995

69. Osswald L, Saller BS, Unger S, De Feo D, Vinnakota JM, Konantz M, et al. Oncogenic Kras G12D causes myeloproliferation via NLRP3 inflammasome activation. Nat Commun (2020) 11(1):1-17. doi: 10.1038/s41467-02015497-1

70. Allam R, Lawlor KE, Yu ECW, Mildenhall AL, Moujalled DM, Lewis RS, et al. Mitochondrial apoptosis is dispensable for NLRP3 inflammasome activation but non-apoptotic caspase-8 is required for inflammasome priming. EMBO Rep (2014) 15(9):982-90. doi: 10.15252/embr.201438463

71. Gurung P, Anand PK, Malireddi RS, Walle LV, Van Opdenbosch N, Dillon $\mathrm{CP}$, et al. FADD and caspase- 8 mediate priming and activation of the 
canonical and noncanonical Nlrp3 inflammasomes. J Immunol (2014) 192 (4):1835-46. doi: 10.4049/jimmunol.1302839

72. Tarassishin L, Lim J, Weatherly DB, Angeletti RH, Lee SC. Interleukin-1induced changes in the glioblastoma secretome suggest its role in tumor progression. J Proteomics (2014) 99:152-68. doi: 10.1016/ j.jprot.2014.01.024

73. Rubartelli A, Bajetto A, Allavena G, Cozzolino F, Sitia R. Post-translational regulation of interleukin $1 \beta$ secretion. Cytokine (1993) 5(2):117-24. doi: 10.1016/1043-4666(93)90050-F

74. Tassi S, Carta S, Delfino L, Caorsi R, Martini A, Gattorno M, et al. Altered redox state of monocytes from cryopyrin-associated periodic syndromes causes accelerated IL-1 $\beta$ secretion. Proc Natl Acad Sci (2010) 107(21):978994. doi: $10.1073 /$ pnas.1000779107

75. Carta S, Tassi S, Pettinati I, Delfino L, Dinarello CA, Rubartelli A. The rate of interleukin- $1 \beta$ secretion in different myeloid cells varies with the extent of redox response to Toll-like receptor triggering. J Biol Chem (2011) 286 (31):27069-80. doi: 10.1074/jbc.M110.203398

76. Perregaux DG, Laliberte RE, Gabel CA. Human monocyte interleukin-1 $\beta$ posttranslational processing Evidence Of A Volume-Regulated Response. J Biol Chem (1996) 271(47):29830-8. doi: 10.1074/jbc.271.47.29830

77. Pelegrin P, Surprenant A. Dynamics of macrophage polarization reveal new mechanism to inhibit IL-1 $\beta$ release through pyrophosphates. EMBO J (2009) 28(14):2114-27. doi: 10.1038/emboj.2009.163

78. Katsnelson MA, Rucker LG, Russo HM, Dubyak GR. K+ efflux agonists induce NLRP3 inflammasome activation independently of Ca2+ signaling. J Immunol (2015) 194(8):3937-52. doi: 10.4049/jimmunol.1402658

79. Zhivotovsky B, Orrenius S. Calcium and cell death mechanisms: a perspective from the cell death community. Cell Calcium (2011) 50 (3):211-21. doi: 10.1016/j.ceca.2011.03.003

80. Yang Y, Jiang G, Zhang P, Fan J. Programmed cell death and its role in inflammation. Mil Med Res (2015) 2(1):12. doi: 10.1186/s40779-015-0039-0

81. Bergsbaken T, Fink SL, Cookson BT. Pyroptosis: host cell death and inflammation. Nat Rev Microbiol (2009) 7(2):99. doi: 10.1038/nrmicro2070

82. Vander Heiden MG, Chandel NS, Schumacker PT, Thompson CB. Bcl-xL prevents cell death following growth factor withdrawal by facilitating mitochondrial ATP/ADP exchange. Mol Cell (1999) 3(2):159-67. doi: 10.1016/S1097-2765(00)80307-X

83. Heerdt BG, Houston MA, Mariadason JM, Augenlicht LH. Dissociation of staurosporine-induced apoptosis from G2-M arrest in SW620 human colonic carcinoma cells: initiation of the apoptotic cascade is associated with elevation of the mitochondrial membrane potential $(\Delta \Psi \mathrm{m})$. Cancer Res (2000) 60(23):6704-13.

84. Wang Y, Shi P, Chen Q, Huang Z, Zou D, Zhang J, et al. Mitochondrial ROS promote macrophage pyroptosis by inducing GSDMD oxidation. J Mol Cell Biol (2019) 11(12):1069-82. doi: 10.1093/jmcb/mjz020

85. Li Y, Wang L, Pappan L, Galliher-Beckley A, Shi J. IL-1 $\beta$ promotes stemness and invasiveness of colon cancer cells through Zeb1 activation. Mol Cancer (2012) 11(1):87. doi: 10.1186/1476-4598-11-87

86. Kapałczyńska M, Kolenda T, Przybyła W, Zajączkowska M, Teresiak A, Filas V, et al. $2 \mathrm{D}$ and $3 \mathrm{D}$ cell cultures-a comparison of different types of cancer cell cultures. Arch Med Sci: AMS (2018) 14(4):910. doi: 10.5114/aoms.2016.63743

87. Lee J, Cuddihy MJ, Kotov NA. Three-dimensional cell culture matrices: state of the art. Tissue Eng Part B: Rev (2008) 14(1):61-86. doi: 10.1089/ teb.2007.0150

88. Najafi M, Farhood B, Mortezaee K, Kharazinejad E, Majidpoor J, Ahadi R. Hypoxia in solid tumors: a key promoter of cancer stem cell (CSC) resistance. J Cancer Res Clin Oncol (2020) 146(1):19-31. doi: 10.1007/ s00432-019-03080-1

89. Sun W, Depping R, Jelkmann W. Interleukin-1 $\beta$ promotes hypoxia-induced apoptosis of glioblastoma cells by inhibiting hypoxia-inducible factor-1 mediated adrenomedullin production. Cell Death Dis (2014) 5(1):e1020-e. doi: 10.1038/cddis.2013.562

90. Shi J, Zhao Y, Wang K, Shi X, Wang Y, Huang H, et al. Cleavage of GSDMD by inflammatory caspases determines pyroptotic cell death. Nature (2015) 526(7575):660. doi: 10.1038/nature15514

91. Wang S, Yuan Y-H, Chen N-H, Wang H-B. The mechanisms of NLRP3 inflammasome/pyroptosis activation and their role in Parkinson's disease. Int Immunopharmacol (2019) 67:458-64. doi: 10.1016/j.intimp.2018.12.019
92. Walle LV, Lamkanfi M. Pyroptosis. Curr Biol (2016) 26(13):R568-R72. doi: 10.1016/j.cub.2016.02.019

93. Schroder K, Sagulenko V, Zamoshnikova A, Richards AA, Cridland JA, Irvine $\mathrm{KM}$, et al. Acute lipopolysaccharide priming boosts inflammasome activation independently of inflammasome sensor induction. Immunobiology (2012) 217(12):1325-9. doi: 10.1016/j.imbio.2012.07.020

94. Paul WE. Interleukin-4: a prototypic immunoregulatory lymphokine. Blood (1991) 77(9):1859-70. doi: 10.1182/blood.V77.9.1859.bloodjournal7791859

95. Pouliot P, Turmel V, Gelinas E, Laviolette M, Bissonnette E. Interleukin-4 production by human alveolar macrophages. Clin Exp Allergy (2005) 35 (6):804-10. doi: 10.1111/j.1365-2222.2005.02246.x

96. Gioannini TL, Teghanemt A, Zhang D, Coussens NP, Dockstader W, Ramaswamy $\mathrm{S}$, et al. Isolation of an endotoxin-MD-2 complex that produces Toll-like receptor 4-dependent cell activation at picomolar concentrations. Proc Natl Acad Sci (2004) 101(12):4186-91. doi: 10.1073/ pnas. 0306906101

97. Kent LW, Rahemtulla F, Hockett RDJr., Gilleland RC, Michalek SM. Effect of lipopolysaccharide and inflammatory cytokines on interleukin- 6 production by healthy human gingival fibroblasts. Infect Immun (1998) 66(2):608-14. doi: 10.1128/IAI.66.2.608-614.1998

98. Lucey DR, Clerici M, Shearer GM. Type 1 and type 2 cytokine dysregulation in human infectious, neoplastic, and inflammatory diseases. Clin Microbiol Rev (1996) 9(4):532-62. doi: 10.1128/CMR.9.4.532

99. Guzzo C, Ayer A, Basta S, Banfield BW, Gee K. IL-27 enhances LPS-induced proinflammatory cytokine production via upregulation of TLR4 expression and signaling in human monocytes. J Immunol (2012) 188(2):864-73. doi: 10.4049/jimmunol.1101912

100. El Chartouni C, Rehli M. Comprehensive analysis of TLR4-induced transcriptional responses in interleukin 4-primed mouse macrophages. Immunobiology (2010) 215(9-10):780-7. doi: 10.1016/j.imbio.2010.05.032

101. Lang T, Lee JP, Elgass K, Pinar AA, Tate MD, Aitken EH, et al. Macrophage migration inhibitory factor is required for NLRP3 inflammasome activation. Nat Commun (2018) 9(1):2223. doi: 10.1038/s41467-018-04581-2

102. Mortaz E, Henricks PA, Kraneveld AD, Givi ME, Garssen J, Folkerts G. Cigarette smoke induces the release of CXCL-8 from human bronchial epithelial cells via TLRs and induction of the inflammasome. Biochim Biophys Acta (2011) 1812(9):1104-10. doi: 10.1016/j.bbadis.2011.06.002.

103. Liao Y, Hussain T, Liu C, Cui Y, Wang J, Yao J, et al. Endoplasmic reticulum stress induces macrophages to produce il- $1 \beta$ during mycobacterium bovis infection via a positive feedback loop between mitochondrial damage and inflammasome activation. Front Immunol (2019) 10:268. doi: 10.3389/ fimmu.2019.00268

104. Luksch H, Schlipfenbacher V, Köhler S, Münch F, Winkler S, Schulze F, et al. Expression of Caspase-1 variants induced ER stress. Pediatr Rheumatol (2015) 13(1):1-. doi: 10.1186/1546-0096-13-S1-P17

105. Lin JH, Walter P, Yen TB. Endoplasmic reticulum stress in disease pathogenesis. Annu Rev Pathol Mech Dis (2008) 3:399-425. doi: 10.1146/ annurev.pathmechdis.3.121806.151434

106. Manousou P, Kolios G, Valatas V, Drygiannakis I, Bourikas L, Pyrovolaki K, et al. Increased expression of chemokine receptor CCR3 and its ligands in ulcerative colitis: the role of colonic epithelial cells in in vitro studies. Clin Exp Immunol (2010) 162(2):337-47. doi: 10.1111/j.1365-2249.2010.04248.x

107. Günther C, Wozel G, Meurer M, Pfeiffer C. Up-regulation of CCL11 and CCL26 is associated with activated eosinophils in bullous pemphigoid. Clin Exp Immunol (2011) 166(2):145-53. doi: 10.1111/j.13652249.2011.04464.x

108. Burke-Gaffney A, Hellewell PG. Eotaxin stimulates eosinophil adhesion to human lung microvascular endothelial cells. Biochem Biophys Res Commun (1996) 227(1):35-40. doi: 10.1006/bbrc.1996.1463

109. Efraim AHNB, Levi-Schaffer F. Roles of eosinophils in the modulation of angiogenesis. Angiogenesis Lymphangiogenesis Clin Implications (2014) 99:138-54. Karger Publishers. doi: 10.1159/000353251

110. Jin L, Liu W-R, Tian M-X, Jiang X-F, Wang H, Zhou P-Y, et al. CCL24 contributes to HCC malignancy via RhoB-VEGFA-VEGFR2 angiogenesis pathway and indicates poor prognosis. Oncotarget (2017) 8(3):5135. doi: 10.18632/oncotarget.14095

111. Lopez-Pastrana J, Ferrer LM, Li Y-F, Xiong X, Xi H, Cueto R, et al. Inhibition of caspase- 1 activation in endothelial cells improves angiogenesis a NOVEL 
THERAPEUTIC POTENTIAL FOR ISCHEMIA. J Biol Chem (2015) 290 (28):17485-94. doi: 10.1074/jbc.M115.641191

Conflict of Interest: The authors declare that the research was conducted in the absence of any commercial or financial relationships that could be construed as a potential conflict of interest.
Copyright (C) 2021 Tezcan, Garanina, Alsaadi, Gilazieva, Martinova, Markelova, Arkhipova, Hamza, McIntyre, Rizvanov and Khaiboullina. This is an open-access article distributed under the terms of the Creative Commons Attribution License (CC BY). The use, distribution or reproduction in other forums is permitted, provided the original author(s) and the copyright owner(s) are credited and that the original publication in this journal is cited, in accordance with accepted academic practice. No use, distribution or reproduction is permitted which does not comply with these terms. 